\title{
Volatility in Equilibrium: Asymmetries and Dynamic Dependencies*
}

\author{
TIM BOLLERSLEV ${ }^{1}$, NATALIA SIZOVA ${ }^{2}$ and GEORGE TAUCHEN ${ }^{3}$ \\ ${ }^{1}$ Duke University, NBER, and CREATES, ${ }^{2}$ Rice University, and ${ }^{3}$ Duke University
}

\begin{abstract}
Stock market volatility clusters in time, appears fractionally integrated, carries a risk premium, and exhibits asymmetric leverage effects. At the same time, the volatility risk premium, defined by the difference between the risk-neutral and objective expectations of the volatility, features short memory. This paper develops the first internally consistent equilibrium-based explanation for all these empirical facts. Using newly available high-frequency intraday data for the S\&P 500 and the VIX volatility index, the authors show that the qualitative implications from the new theoretical continuous-time model match remarkably well with the distinct shapes and patterns in the sample autocorrelations and dynamic cross-correlations actually observed in the data.
\end{abstract}

JEL Classification: C22, C51, C52, G12, G13, G14

\section{Introduction}

Modeling and forecasting of stock market return volatility has received unprecedented attention in the academic literature over the past two decades. The three most striking empirical regularities to emerge from this burgeoning literature arguably concern: (i) the highly persistent own dynamic dependencies in the volatility, ${ }^{1}$ (ii) the existence of a typically positive volatility risk premium as manifested by the variance swap rate exceeding the corresponding expected future volatility, $^{2}$ (iii) the apparent asymmetry in the lead-lag relationship between returns

\footnotetext{
* We would like to thank Bernard Dumas (the Editor), an anonymous referee, Torben G. Andersen, Laurent Calvet, Niklas Wagner, and participants in the 2008 Volatility Symposium at Center for Research in Econometric Analysis of Time Series (CREATES), University of Aarhus, Denmark, the 2009 Financial Econometrics Conference at Toulouse University, the 2010 SoFiE Conference in Melbourne, along with several university seminars for helpful comments and discussion. Bollerslev's work was supported by a grant from the National Science Foundation (NSF) to the National Bureau of Economic Research (NBER) and Center for Research in Econometric Analysis of Time Series (CREATES) funded by the Danish National Research Foundation.

1 The historically low volatility in the years preceding the Fall 2008 financial crises and the subsequent sustained heightened volatility provide anecdotal evidence for this idea.

2 The preponderance of options traders "selling" volatility to gain the premium indirectly supports the notion of volatility carrying a risk premium.
} 
and volatility. ${ }^{3}$ Despite these now well-documented and generally accepted empirical facts, no formal theoretical model yet exists for explaining all these features within a coherent economic framework. This paper fills that void by developing an entirely self-contained equilibrium-based explanation for the observed asymmetry and volatility risk premium. The model is based on the Epstein-Zin-Weil recursive preference structure and is cast in continuous time, thereby allowing for a direct assessment of its ability to match the qualitative features of the data across different sampling frequencies, including intraday cross-correlation patterns as well as longer run dynamic dependencies.

The specific contributions of the paper are two-fold. First, from an empirical perspective, we use ultra high-frequency 5 -min data on both returns and volatility. Our use of the Chicago Board Options Exchange (CBOE) VIX volatility index, constructed to match to the risk-neutral expectation of the forward integrated variance, together with the actual S\&P 500 index affords a much sharper view of the dynamic asymmetries and cross-correlations between returns and volatility than hitherto available in the literature. ${ }^{4}$ Intuitively, the higher resolution traces directly to the fact that correlations are second moment statistics, which are well known to be much more accurately determined from intraday high-frequency, as opposed to say daily, data. Second, from a theoretical perspective, we set forth a continuous-time model, albeit highly stylized, that uses the shadow prices implied by an optimizing economic agent operating within an endowment environment to help understand the three striking regularities noted above - hence, the term "equilibrium." The model fills the above-mentioned void in the literature and stands in direct contrast to the less formal "reduced-form" explanations proffered for some of the observed regularities, most notably those associated with the so-called "leverage effect" first observed by Black (1976) and Christie (1982). Working in continuous time presents a number of new theoretical challenges but is essential to avoid internal timing inconsistencies in regard to the dynamic dependencies in the VIX, which is formally defined as square root of the expectation of the continuously integrated forward variance. Even though our economic, or "equilibrium," model is too stylized to be directly estimable, its general qualitative

\footnotetext{
3 Again, the heightened volatility following Russia's default and the LTCM debacle in September 1998 , the relatively low volatility accompanying the rapid run-up in prices during the tech bubble, as well as the recent sharp increase in volatility accompanying the Fall 2008 financial crises and sharp market declines are all in line with this asymmetry.

4 To the best of our knowledge, no clear depictions of the dynamic cross-correlations, such those in Figure 2 below, currently exists in the literature. Using 5-min returns alone, Bollerslev, Litvinova, and Tauchen (2006) have previously adduced a negative relationship between the magnitude and the sign of contemporaneous and lagged returns. Unlike the VIX ${ }^{2}$, however, the absolute returns provide extremely noisy measures of the local volatility and, in turn, do not afford a clear picture of the forwardpositive relationship essential to the risk-based explanation of the present paper.
} 
predictions are rich enough to be compared with the documented empirical patterns, thus making the model and the basic underlying economic mechanisms refutable.

Before discussing the model any further, it is instructive to illustrate the new empirical regularities that we seek to explain. To that end, the top most solid line in Figure 1 shows the sample autocorrelations for the aggregate market volatility out to a lag length of 90 days. The calculations are based on daily data for the squared option-implied volatility index VIX over the past two decades; further details concerning the data and different volatility measures are given in Section 4. The autocorrelations in Figure 1 decay at a very slow rate and remain numerically large and statistically significant for all lags. Consistent with these highly persistent own dynamic dependencies in the volatility, it is now widely accepted that the typical rate of decay is so slow as to be best described by a fractionally integrated long memory-type process; for some of the earliest empirical evidence along these lines, see, for example, Robinson (1991), Ding, Grange, and Engle (1993), and Baillie, Bollerslev, and Mikkelsen (1996).

The VIX index in effect represents the market's expectation of the cumulative variation of the S\&P 500 index over the next month plus any premium for bearing the corresponding volatility risk. ${ }^{5}$ Isolating the variance risk premium, the second line in Figure 1 shows the daily autocorrelations for the difference between the squared VIX index and the 1 month ahead forecasts from a simple reduced-form time series model for the actually observed daily realized variation in the S\&P 500 index; further details concerning the high frequency-based realized volatility series and the construction of the model forecasts are again deferred to Section 4. Although the autocorrelations still indicate positive own dynamic dependencies for up to several weeks, the premium is clearly not as persistent as the volatility process itself. Again, this is not a new empirical result per se. For instance, the analysis in Bollerslev, Bollerslev, and Mikkelsen (2011) also supports the idea of relatively fast mean reversion in the volatility risk premium, as does the empirical evidence of fractional cointegration between implied and realized volatility presented by, for example, Bandi and Perron (2006) and Nielsen (2007). ${ }^{6}$

Next, in order to highlight the aforementioned return-volatility asymmetry, the first panel in Figure 2 plots the cross-correlations between leads and lags of the S\&P

\footnotetext{
$\overline{5}$ The variance risk premium is formally defined as the difference between the expected future variation under the risk-neutral and actual probability measures.

${ }^{6}$ As noted by a referee, the lower persistence in the variance premium could at least in part be due to an errors-in-variables-type problem created by the use of an estimated forecast proxy in place of the true population conditional expectation for the squared VIX. However, on implementing the instrumental variables technique recently developed by Hansen and Lunde (2010) to account for this problem, we find that the robust to measurement errors autocorrelations differ little from those shown in Figure 1. Further details concerning these results are available in supplementary material.
} 


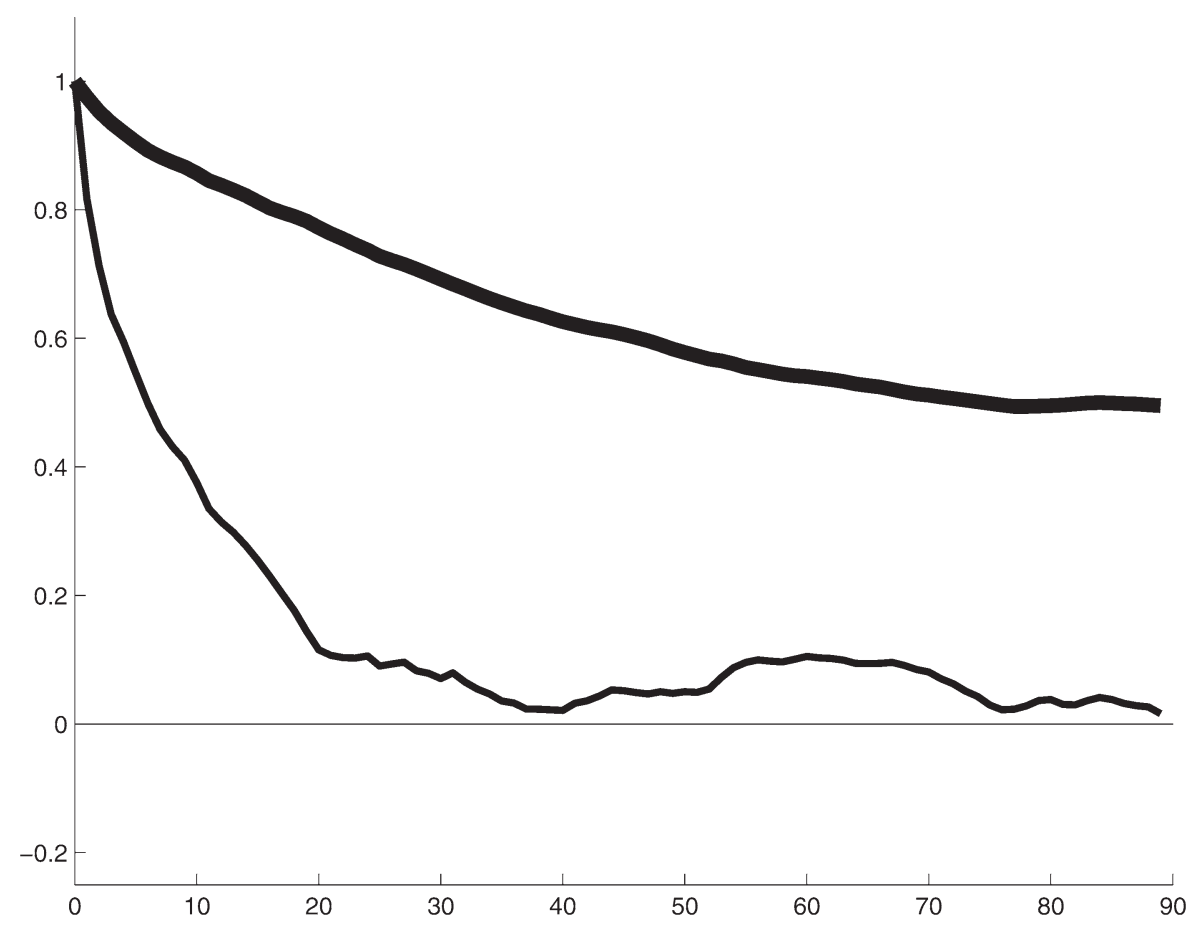

Figure 1. Sample autocorrelations. The top most solid line shows the sample autocorrelations for the VIX2 volatility index to a lag length of 90 days. The lower line shows the sample autocorrelations for the variance risk premium. The calculations are based on daily data and variable definitions as described in more detail in Section 4.1.

500 returns and the squared option-implied VIX volatility index. ${ }^{7}$ Bollerslev, Litvinova, and Tauchen (2006) have previously demonstrated the advantage of using high-frequency intraday returns for more effectively estimating and analyzing the lead-lag relationship between returns and volatility using the absolute returns as a proxy for the latent spot volatility. We follow their lead in the use of highfrequency 5 -min observations. However, instead of proxying the volatility by the absolute returns, we rely on actual observations on the S\&P 500 returns and the VIX volatility index recorded at a 5-min sampling frequency in calculating the sample cross-correlations for leads and lags ranging up to 22 days or 1,716 leads

\footnotetext{
$\overline{7}$ Note that Figure 2 shows cross-correlations between the levels of the variance-related variables because the expected part of future returns, reflected in the right-hand side of the plots, depends on their levels, not the first differences or innovations. Both variance-related variables are stationary in levels as seen from Figure 1.
} 

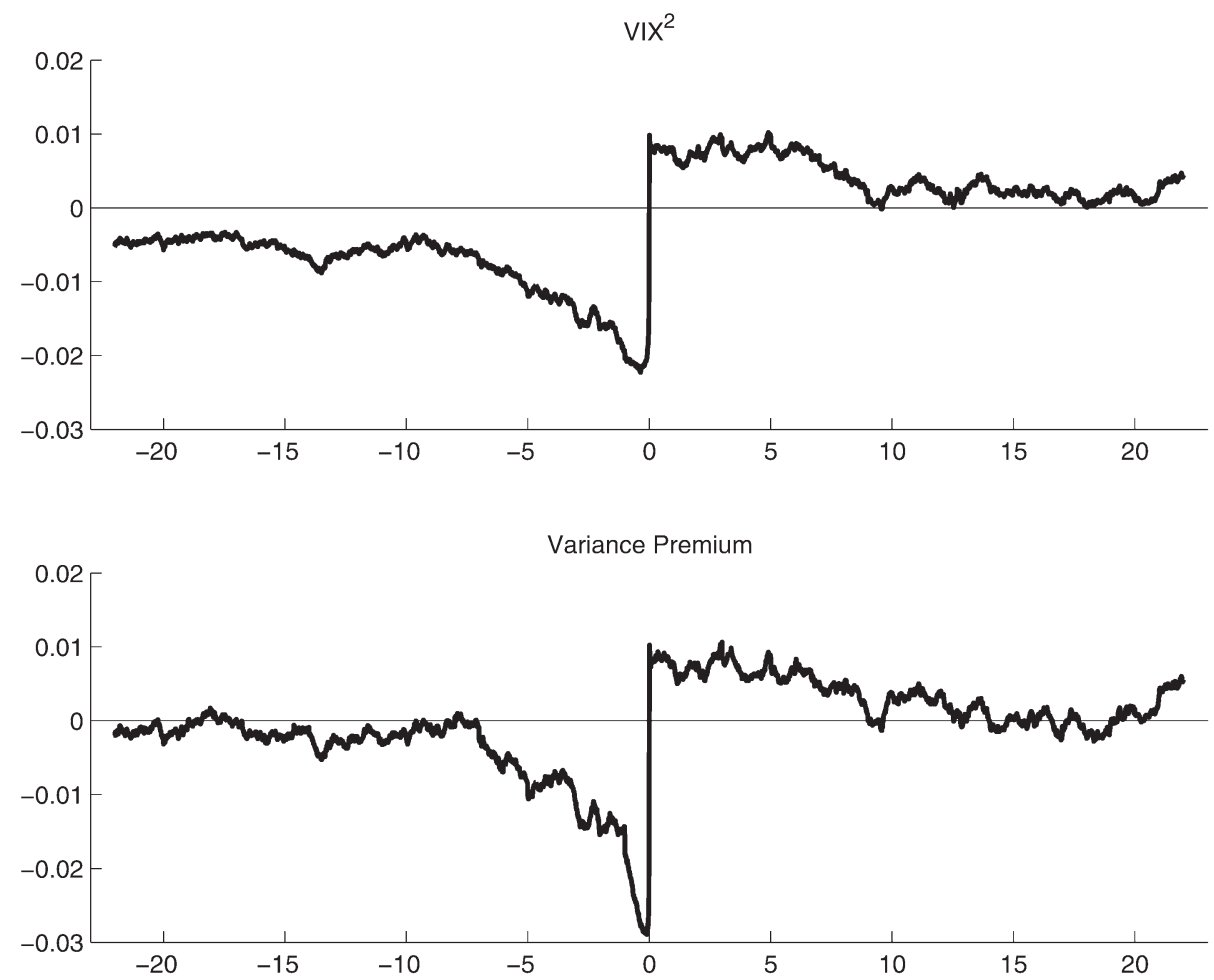

Figure 2. Sample cross-correlations. The top panel shows the sample cross-autocorrelations between the VIX ${ }^{2}$ and lags and leads of the returns ranging from -22 to 22 days. The bottom panel shows the sample cross-autocorrelations between the variance risk premium and the returns. The calculations are based on high-frequency 5-min data and variable definitions as further detailed in Section 4.1.

and lags at the 5-min sampling. High-frequency data for the VIX have only recently become available, so that the cross-autocorrelations depicted in Figure 2 are necessarily based on a shorter 5-year calendar-time span compared with the longer 18year sample of daily observations used for illustrating the own dynamic dependencies in the previous Figure 1. Nonetheless, the use of high-frequency data over this shorter sample still reveals a striking negative pattern in the correlations between the volatility and the lagged returns, lasting for several days. On the other hand, the correlations between the volatility and the future returns are all positive, albeit closer to zero.

This systematic pattern in the high frequency-based cross-correlations between returns and volatility is directly in line with the empirical evidence from numerous studies based on coarser lower frequency daily data and specific parametric models, including the early influential work by French, Schwert, and Stambaugh (1987), Schwert (1990), Nelson (1991), Glosten, Jagannathan, and Runkle (1993), and 

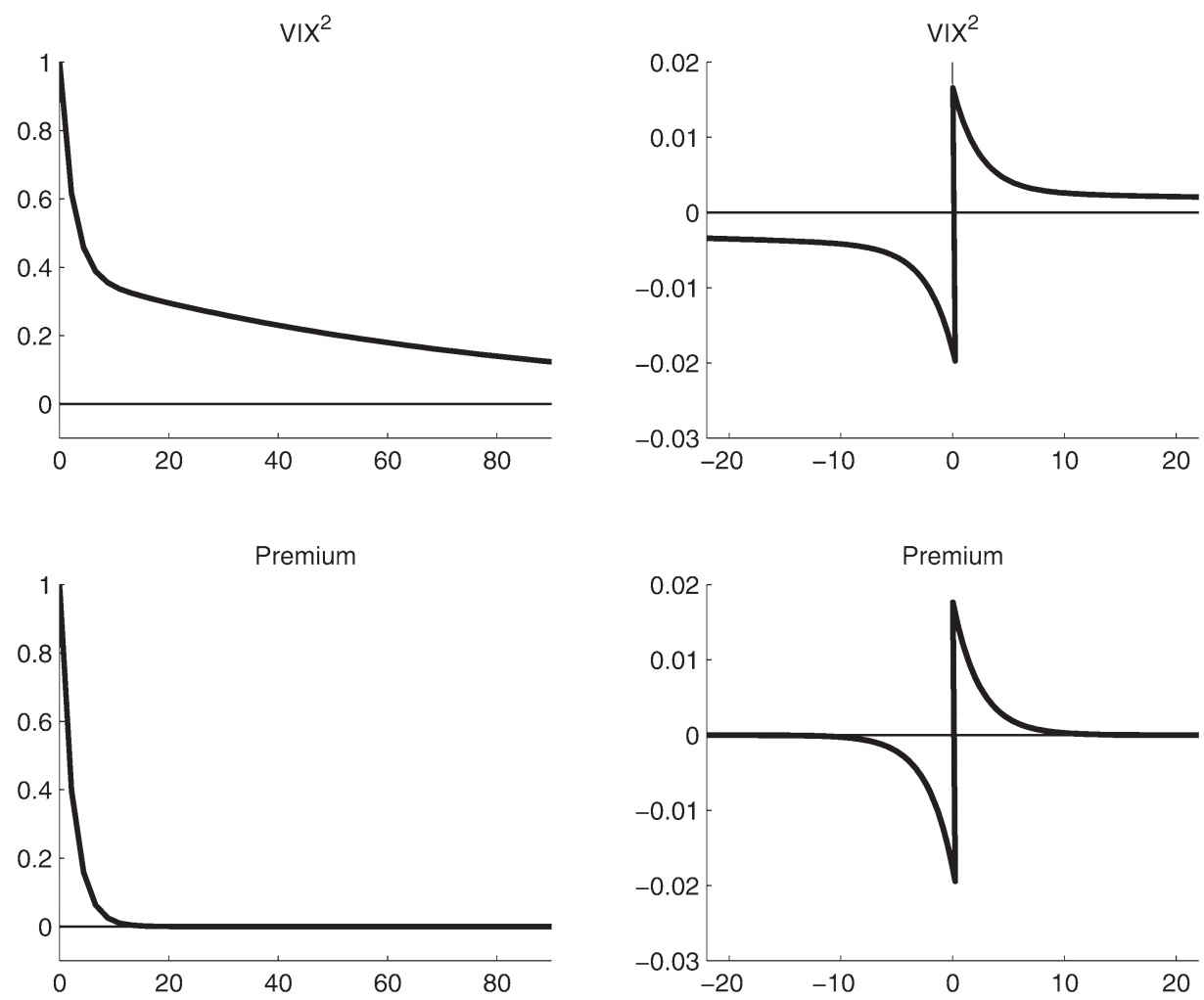

Figure 3. Calibrated autocorrelations and cross-correlations. The plots are based on the model calibrated in Appendix C. The two left-hand panels show the model-implied autocorrelations for the VIX ${ }^{2}$ volatility index and the variance risk premium to a lag length of 90 days. The right-hand panels show the model-implied cross-correlations between the VIX ${ }^{2}$ volatility index and the variance risk premium to a lag length of 22 days.

Campbell and Hentschell (1992). Also, following Black (1976), the left part of Figure 2 and the negative correlations between lagged returns and current volatility has now commonly referred to in the literature as a "leverage effect," while the right part of the figure and the positive correlations between current volatility and future returns have been termed a "volatility feedback effect." 8

Further relating our empirical findings to the existing literature, it is worth noting that while numerically small compared to the estimates reported in some of the

\footnotetext{
$\overline{8}$ It is now widely agreed that the negative correlations between lagged returns and current volatility have little if anything to do with changes in financial leverage (see, e.g., Figlewski and Wang, 2002). In fact, the two effects may be viewed as flip sides of the same coin. Quoting from Campbell, Lo, and MacKinlay (1997, chapter 12): "If expected stock returns increases when volatility increases, and if expected dividends are unchanged, then stock prices should fall when volatility increases."
} 

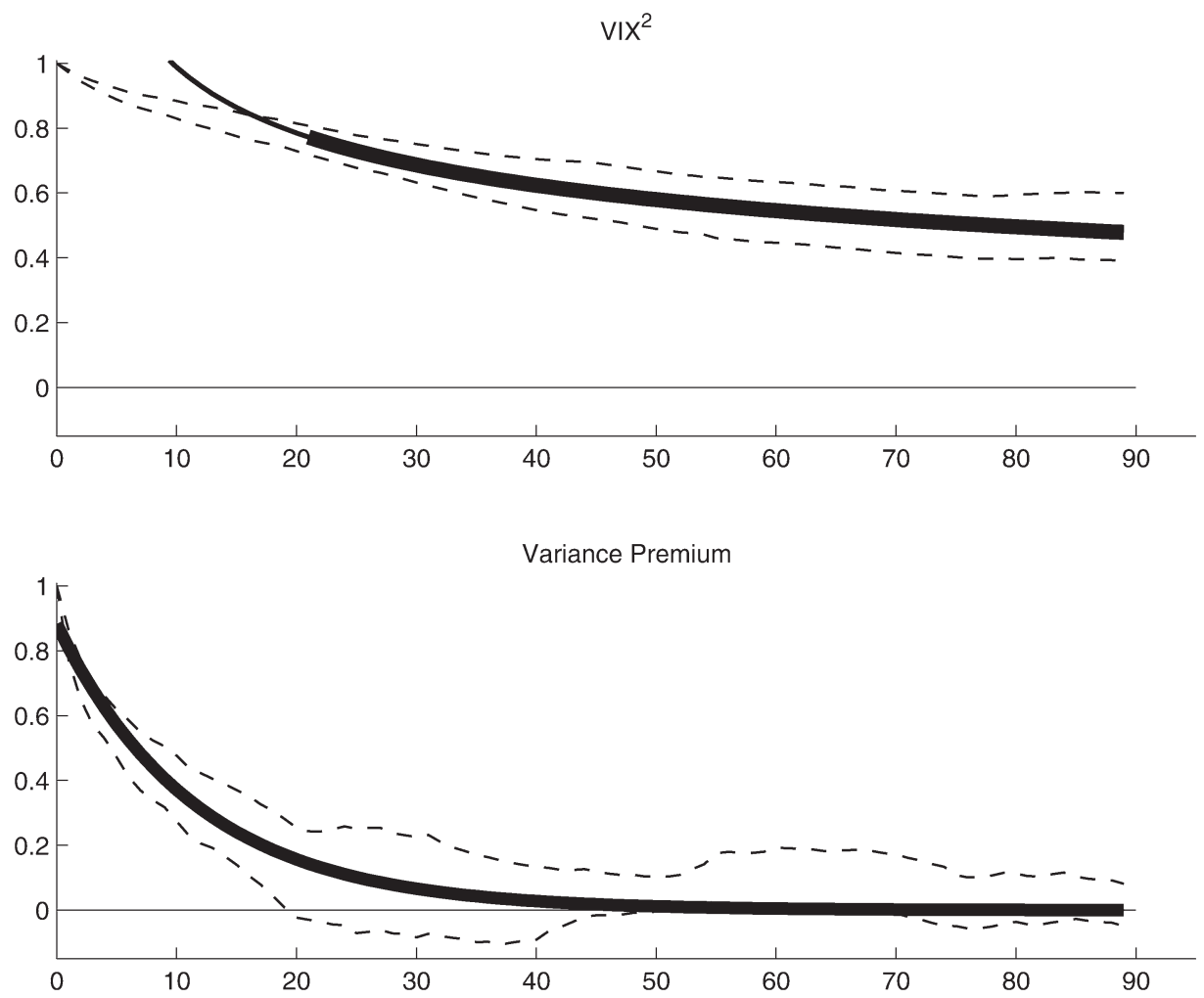

Figure 4. Model-implied autocorrelations. The top panel shows the autocorrelations for the VIX ${ }^{2}$ volatility index to a lag length of 90 days. The solid line gives the model-implied autocorrelations under the assumption of long memory in the underlying fundamental volatility process. The bottom panel shows the autocorrelations for the variance risk premium $v p$. The solid line gives the modelimplied autocorrelations. The pair of dashed lines included in both panels represents $95 \%$ confidence intervals for the corresponding sample autocorrelations based on daily data from 1990 through 2007.

above-mentioned studies based on coarser daily data, the magnitudes of the crosscorrelations in Figure 2 are frequency dependent. As such, they only appear misleadingly low. For instance, taking into account the differences in sampling frequency, the -0.025 correlation of the squared VIX with the contemporaneous 5 -min return translates into a correlation of roughly -0.20 with daily returns.

Taking the analysis one step further, the bottom panel in Figure 2 shows the cross-correlations between the 5-min S\&P 500 returns and a more modern volatility-type measure, the variance risk premium, where as before the variance risk premium is defined as the difference between the squared VIX index and the corresponding forecast constructed from a simple reduced-form time series model for the daily realized volatilities. Comparing this plot to the return-volatility 

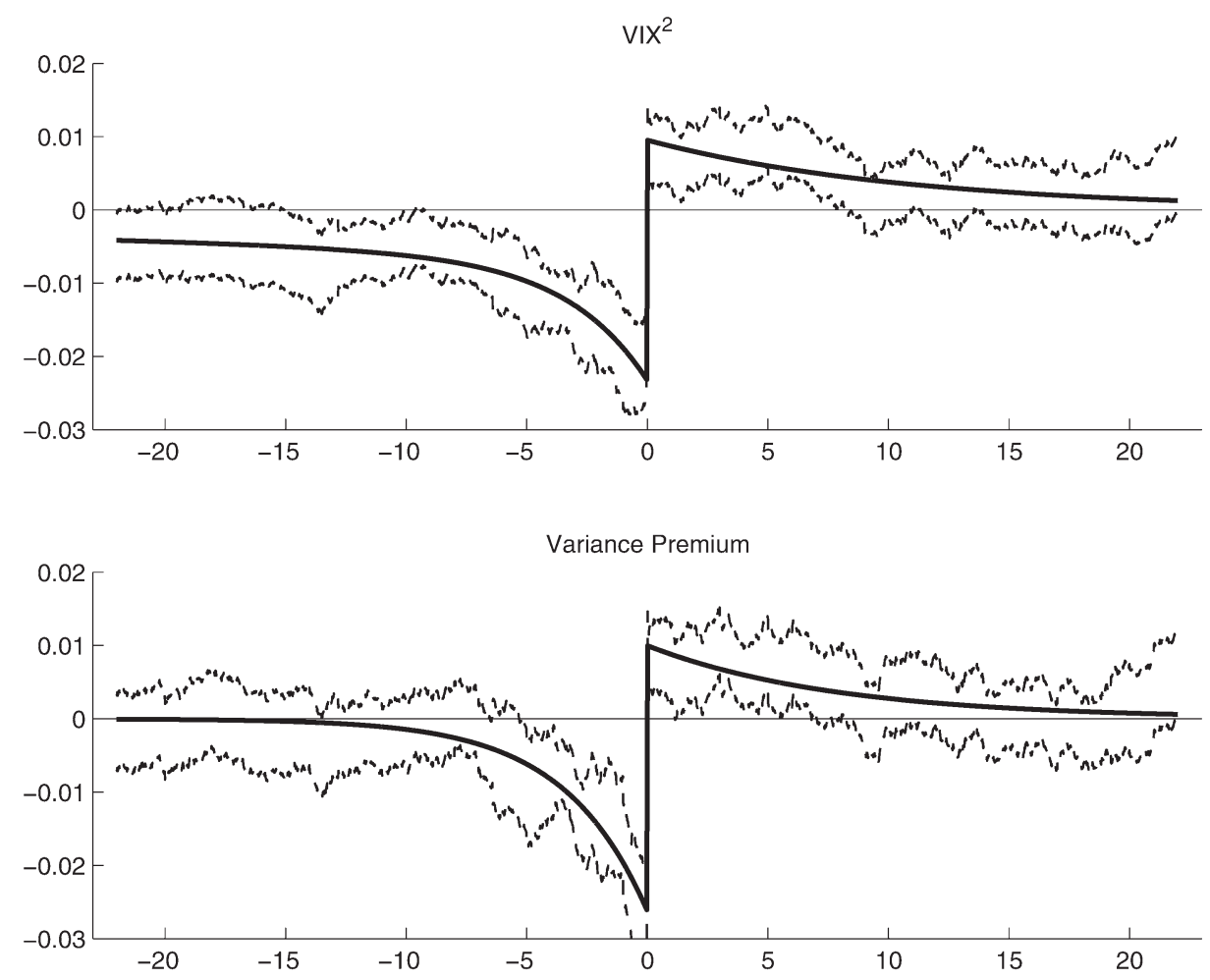

Figure 5. Model-implied cross-correlations. The top panel shows the sample cross-autocorrelations between the VIX ${ }^{2}$ volatility index and lags and leads of the returns ranging from -22 to 22 days. The bottom panel shows the cross-correlations between the variance risk premium $v p$ and the returns. The solid lines give the cross-correlations implied by the theoretical model. The pair of dashed lines represents $95 \%$ confidence intervals for the corresponding sample cross-correlations based on high-frequency 5-min observations from 2003 through 2007.

dependencies in the top panel, the signs of the cross-correlations generally coincide. However, there is a noticeably faster decay toward zero in the magnitude of the cross-correlations between the variance risk premium and the lagged returns compared to the decay in the cross-correlations between the squared VIX index itself and the lagged returns. ${ }^{9}$ This difference closely mirrors the difference in the shape and the rate of decay in the standard sample autocorrelations for the two daily volatility series depicted in Figure 1.

The key empirical return-volatility patterns and dynamic dependencies illustrated in the two figures are consistent with the idea that volatility carries a risk

\footnotetext{
$\overline{9}$ Bollerslev and Zhou (2006) have previously noted that the return-volatility asymmetry tend to be stronger for implied than for realized volatilities.
} 


\section{Table I. Summary Statistics}

The table reports summary statistics for continuously compounded returns $r_{t}$, implied variances VIX $\mathrm{X}_{t}^{2}$, monthly realized variances $\mathrm{RV}_{t, t+22}$, and the variance risk premium $v p_{t}=\mathrm{VIX}_{t}^{2}-\widehat{E} \mathrm{RV}_{t, t+22}$. The realized variances are constructed from the summation of high-frequency 5 -min squared returns. The expectations for the future variances $\widehat{E}_{t} \mathrm{RV}_{t, t+22}$ are based on the HAR-RV forecasting model discussed in the text. All the variables are in percentage form. The daily data extend from January 2 1990 to October 31 2007. The 5-min sample spans September 222003 to August 312007.

\begin{tabular}{lrrrr}
\hline & \multicolumn{1}{c}{$r_{t}$} & $\mathrm{VIX}_{t}^{2}$ & $\mathrm{RV}_{t, t}+22$ & $\widehat{v} p_{t}$ \\
\hline Daily sampling (1990-2007) & & & & \\
$\quad$ Mean & 11.14 & 32.81 & 23.74 & 8.96 \\
Standard deviation & 15.67 & 23.70 & 24.12 & 12.62 \\
Skewness & -0.05 & 1.88 & 2.59 & -1.86 \\
$\quad$ Excess kurtosis & 3.75 & 4.66 & 8.01 & 17.53 \\
Five-min sampling (2003-07) & & & & \\
Mean & 8.96 & 17.48 & 11.28 & 6.17 \\
Standard deviation & 11.31 & 8.40 & 7.68 & 5.25 \\
Skewness & 0.70 & 2.90 & 3.81 & 1.94 \\
Excess kurtosis & 42.12 & 15.12 & 17.90 & 9.40 \\
\hline
\end{tabular}

premium. Standard equilibrium-based models build around a representative agent with time-separable utility rules out priced volatility risk. Instead, following the literature on so-called long-run risk models pioneered by Bansal and Yaron (2004), we will here assume a representative agent with Epstein-Zin-Weil preferences, tantamount to a preference for early resolution of uncertainty. Our model is cast in continuous time, thereby avoiding any assumptions about the decision interval of the agent. The Epstein-Zin-Weil preference structure was first employed in a continuous-time asset pricing setting by Duffie and Epstein (1992a). In this situation the stochastic discount factor (SDF) will depend not only on the consumption growth rate but also on the future investment opportunities. ${ }^{10}$ Consequently, the aggregate market return will be a function of the expected growth in the economy, as in the traditional time-separable utility case, as well as the uncertainty about the future economic growth (see, e.g., Campbell, 1996). Intuitively, this explains why investors may be willing to pay an uncertainty premium, and in turn why the VIX may differ from the corresponding actual return volatility, and why the corresponding variance risk premium may act as a separately priced risk factor.

The same mechanism involving time-varying economic uncertainty and a preference for early resolution of uncertainty also underlies the model of Bollerslev, Tauchen, and Zhou (2009), which parallels the present study in allowing the volatility-of-volatility in the economy, or the economic uncertainty, to be

\footnotetext{
${ }_{10}$ In contrast to the expression for the SDF involving the compensator function derived in Duffie and Epstein (1992a), we find it more convenient to work with the discount factor expressed in terms of the consumption growth rate and the market return.
} 
Table II. Calibration Parameters

The table reports the parameter values used in the calibration of the theoretical model discussed in Section B. BYa and BY denote small adjustments to, or values taken directly from, Bansal and Yaron (2004), BST denotes values calibrated by the authors, and K denotes values from Kiku (2008).

\begin{tabular}{ccc}
\hline & Parameter & Source \\
\hline Preferences & & \\
$\rho$ & $-\log (0.999)$ & BYa \\
$\gamma$ & 7.50 & BYa \\
$\psi$ & 2.50 & BYa \\
Consumption growth & & \\
$\mu_{g}$ & 0.0015 & BY \\
$\kappa_{x}$ & $-\log (0.960)$ & BYa \\
$\sigma_{x}$ & $0.044 \sqrt{\mu_{\sigma}}$ & BY \\
Dividend growth & & \\
$\mu_{d}$ & 0.0013 & BYa \\
$\phi_{x}$ & 3.0 & BY \\
$\phi_{\sigma}$ & $4.0 \sqrt{0.55}$ & BYa, K \\
$\phi_{\sigma x}$ & 0.0 & K \\
$\sigma_{d}$ & $4.0 \sqrt{0.45} \mu_{\sigma}^{1 / 2}$ & BYa, K \\
Volatility & & \\
$\mu_{\sigma}$ & $0.0078^{2}$ & BY \\
$\kappa_{\sigma}$ & $-\log (0.760)$ & BST \\
$\mu_{q}$ & $0.350 \times 10^{-6}$ & BST \\
$\kappa_{q}$ & $-\log (0.0001)$ & BST \\
$\varphi_{q}$ & 0.07 & BST \\
\hline
\end{tabular}

determined by its own separate stochastic process. The new equilibrium model developed here is also related to the long-run risk model of Drechsler and Yaron (2011), in which the expected growth rate in consumption and the volatility of consumption growth are both allowed to "jump." 11 The present paper extends both these studies by considering the cross-correlation between volatility and returns at all leads and lags. Of course, as noted above, it has long been recognized from reduced-form analysis (e.g., Campbell, Lo, and MacKinlay, 1997, chapter 12) that the price of volatility risk must be negative, in turn implying a negative contemporaneous correlation between return and volatility. This negative correlation was, to the best of our knowledge, first placed within a structural equilibrium framework based on Epstein-ZinWeil preferences by Bansal and Yaron (2004) (see their Equation (12) and surrounding discussion). With the noticeable exceptions of Bollerslev, Tauchen, and Zhou (2009) and Drechsler and Yaron (2011), however, other structural formulations generally presume overly restrictive dynamics for the evolution of economic uncertainty,

\footnotetext{
$\overline{11}$ A related long-run risk model in which the economic uncertainty, or the volatility of consumption growth, is allowed to "jump" in continuous time has also recently been explored by Eraker (2008), in an attempt to explain the existence of a (on average) positive volatility risk premium.
} 


\section{Table III. Calibration Results}

The column denoted "Observed" refers to the sample values for the relevant moments in the studies indicated in the "Source" column. The corresponding population moments implied by the theoretical model discussed in Section B evaluated at the parameter values given in Table II are reported in the last column denoted "Calibration". $E\left(r_{m, t}-r_{f, t}\right)$ and $\sqrt{\operatorname{Var}\left(r_{m, t}\right)}$ refer to the equity premium and the equity return standard deviation, respectively. For sources, BST denotes values reported in Table I above, BY refers to values taken from Bansal and Yaron (2004), and DY denotes values from Drechsler and Yaron (2011).

\begin{tabular}{|c|c|c|c|}
\hline & Observed & Source & Calibration \\
\hline \multicolumn{4}{|l|}{ System dynamics } \\
\hline \multicolumn{4}{|l|}{ Consumption ${ }^{\mathrm{a}}$} \\
\hline$E\left(g_{t}\right)$ & 1.80 & BY & 1.80 \\
\hline$\sqrt{\operatorname{Var}\left(g_{t}\right)}$ & 2.93 & BY & 2.70 \\
\hline \multicolumn{4}{|l|}{ Dividends ${ }^{\mathrm{a}}$} \\
\hline$E\left(d_{t}\right)$ & 1.54 & DY & 1.54 \\
\hline$\sqrt{\operatorname{Var}\left(d_{t}\right)}$ & 13.69 & DY & 10.81 \\
\hline \multicolumn{4}{|l|}{ Correlations $^{\mathrm{a}}$} \\
\hline $\operatorname{Corr}\left(g_{t}, g_{t-1}\right)$ & 0.43 & DY & 0.18 \\
\hline $\operatorname{Corr}\left(d_{t}, d_{t-1}\right)$ & 0.14 & DY & 0.10 \\
\hline $\operatorname{Corr}\left(d_{t}, g_{t}\right)$ & 0.59 & DY & 0.74 \\
\hline \multicolumn{4}{|l|}{ Model-implied moments } \\
\hline \multicolumn{4}{|l|}{ Returns $^{\mathrm{a}}$} \\
\hline$E\left(r_{f, t}\right)$ & 0.82 & DY & 1.19 \\
\hline$\sqrt{\operatorname{Var}\left(r_{f, t}\right)}$ & 1.89 & DY & 4.43 \\
\hline$E\left(r_{m, t}-r_{f, t}\right)$ & 6.23 & DY & 5.95 \\
\hline$\sqrt{\operatorname{Var}\left(r_{m, t}\right)}$ & 19.37 & DY & 19.17 \\
\hline \multicolumn{4}{|l|}{ Volatility and premium } \\
\hline$E\left(\mathrm{VIX}^{2}\right)$ & 32.81 & BST & 37.87 \\
\hline$E\left(v p_{t}\right)$ & 8.96 & BST & 7.25 \\
\hline
\end{tabular}

${ }^{\mathrm{a}}$ The reported value is appropriately annualized to ease interpretation.

which imply a counterfactual constant variance risk premium that simply cannot explain the rich cross-correlation patterns seen in Figure 2.

Also, in contrast to the discrete-time formulations employed in most previous studies, Bollerslev, Tauchen, and Zhou (2009) and Drechsler and Yaron (2011) both included, the continuous-time formulation adopted here has the distinct advantage of allowing for the calculation of internally consistent model implications across all sampling frequencies and return horizons. Of course, as already noted, the new model developed here also accommodates much richer and empirically realistic longer run volatility dependencies, including the possibility of fractional integration. Moreover, our continuous-time setup permits an internally consistent definition of the risk-neutral expectations and the VIX volatility index, thereby avoiding the inherent problem in discrete-time asset pricing models with Generalized Autoregressive Conditional Heteroskedastic-type errors that the (conditional) variance is known one period in advance and therefore formally cannot generate a variance premium.

The new model developed below is also related to the multifractal approach put forth in the series of papers by Calvet and Fisher $(2007,2008)$. In particular, on 
assuming that the dividend growth volatility follows a multifractal process, as in Calvet and Fisher (2002), along with an Epstein-Zin-Weil-type representative agent, as in the model developed here, the equilibrium models in Calvet and Fisher (2007, 2008) are also able to generate endogenous volatility feedback effects and long memory-type features in the volatility, along with negative skewness in the returns due to the impact of learning. None of these former studies, however, has considered the implications of the multifractal setup and assumptions for the risk-neutral expectation of the volatility as embedded within the VIX, nor the dynamic dependencies in the corresponding volatility risk premium.

Other recent studies concerned with the equilibrium pricing of volatility risk include Gabaix (2010) and Wachter (2010), both of whom analyze the implications of rare disasters, and Lettau, Ludvigson, and Wachter (2009), who emphasize the role of low-frequency movements in macroeconomic uncertainty for explaining lowfrequency multiyear movements in stock market valuations. Several studies more squarely rooted in the option-pricing literature have also explored the equilibrium implications of allowing for richer volatility dynamics and nonstandard preference structures; see, for example, the recent papers by Benzoni, Collin-Dufresne, and Goldstein (2006), Eraker and Shaliastovich (2008), Santa-Clara and Yan (2010), and the references therein.

The empirical focus of the present paper and the use of high-frequency intraday data for the S\&P 500 returns and the VIX are distinctly different from all these previous studies, and to the best of our knowledge, no other coherent economic mechanism for explaining all the dynamic dependencies and asymmetries in the volatility and volatility risk premium depicted in Figures 1 and 2 is yet available in the literature. In order to focus on the volatility channels that we seek to illuminate, the model is deliberately kept as simple as possible and thus would not be expected to "match" all asset pricing moments. Nonetheless, in keeping with the basic setup in Bansal and Yaron (2004), the general modeling framework is flexible enough to allow for a reasonable "match" with many of the more "standard" moments as well.

The plan for the rest of the paper is as follows. The new theoretical model is formally defined and solved in Section 2. This section also briefly discusses a simple calibration for the model designed to "match" some of the more "standard" asset pricing moments. The equilibrium implications from an extended long-memory version of the model in regard to the key return-volatility asymmetries and own dynamic volatility dependencies that we seek to explain are presented in Section 3. The data used in the construction of the figures discussed above and the model's ability to reproduce these basic empirical features are the subject of Section 4. Section 5 concludes. Most of the mathematical proofs are deferred to two appendixes, as are further details concerning the stylized calibration alluded to in Section 2. 


\section{Volatility in Equilibrium}

The classic continuous-time intertemporal capital asset pricing model (CAPM) of Merton (1973) is often used to justify the existence of a volatility risk premium. However, this model is inconsistent with observed long-range volatility dependence as seen in Figure 1 and is incapable of explaining the dynamic leverage effect and asymmetric return-volatility dependencies shown in Figure 2. The continuous-time endowment economy developed here instead builds on the discrete-time long-run risk model pioneered by Bansal and Yaron (2004). We begin by describing an initial continuous-time model setup and solution under short-memory Markov dynamics. We next validate its predictions for standard variables via a simple calibration. We also show how it can explain the dynamic asymmetries but fails on matching longrange volatility dependence. We subsequently show how to adjust the model to incorporate empirically relevant long-memory dependencies while preserving the key return-volatility implications of the initial model.

\subsection{INITIAL MODEL SETUP AND ASSUMPTIONS}

Let the local geometric growth rate of consumption $C_{t}$ in the economy be denoted by $g_{t} \equiv \frac{\mathrm{d} C_{t}}{C_{t}}$, which we assume follows the continuous-time process

$$
g_{t}=\left(\mu_{g}+x_{t}\right) \mathrm{d} t+\sigma_{g, t} \mathrm{~d} W_{t}^{c} .
$$

Here $\mu_{g}$ denotes the constant long-run mean growth rate, $x_{t}$ is the mean-zero stochastic component of consumption growth, $\sigma_{g, t}$ refers to economic uncertainty, that is, the conditional volatility of the growth rate, and $W_{t}^{c}$ is a standard Wiener process. The stochastic growth component follows the standard dynamics

$$
\mathrm{d} x_{t}=-\kappa_{x} x_{t} \mathrm{~d} t+\sigma_{x} \mathrm{~d} W_{t}^{x},
$$

where $\kappa_{x}>0$, and $W_{t}^{x}$ is a standard Wiener process independent of $W_{t}^{c}$. For small $\kappa_{x}$, this is a long-run risk-type specification, but we abstract from stochastic volatility of consumption growth itself. We also assume a dividend asset with dividend growth dynamics $\left(\mathrm{d}_{t}=\mathrm{d} D_{t} / D_{t}\right)$,

$$
\mathrm{d}_{t}=\left(\mu_{d}+\phi_{x} x_{t}\right) \mathrm{d} t+\phi_{\sigma} \sigma_{g, t} \mathrm{~d} W_{t}^{c}+\phi_{\sigma x} \mathrm{~d} W_{t}^{x}+\sigma_{d} \mathrm{~d} W_{t}^{d},
$$

where $\mu_{d}$ refers to the unconditional mean dividend growth rate, the $\phi$ s reflect the dividend's exposures to the consumption risk factors, and the dividend growth innovation volatility $\sigma_{d}$ is assumed to be constant (nonstochastic) for simplicity. Importantly, we assume that the volatility dynamics in the economy are governed by the continuous-time affine processes, 


$$
\begin{aligned}
& \mathrm{d} \sigma_{g, t}^{2}=\kappa_{\sigma}\left(\mu_{\sigma}-\sigma_{t}^{2}\right) \mathrm{d} t+\sqrt{q_{t}} \mathrm{~d} W_{t}^{\sigma}, \\
& \mathrm{d} q_{t}=\kappa_{q}\left(\mu_{q}-q_{t}\right) \mathrm{d} t+\varphi_{q} \sqrt{q_{t}} \mathrm{~d} W_{t}^{q},
\end{aligned}
$$

where the two Wiener processes $W_{t}^{\sigma}$ and $W_{t}^{q}$ are independent and jointly independent of $W_{t}^{c}$ and $W_{t}^{x}$, and the parameters satisfy the nonnegativity restrictions $\mu_{\sigma}>0$, $\mu_{q}>0, \kappa_{\sigma}>0, \kappa_{q}>0$, and $\varphi_{q}>0 .{ }^{12}$ The stochastic volatility process $\sigma_{g, t}^{2}$ represents time-varying economic uncertainty in consumption growth, with the volatility-of-volatility process $q_{t}$ in effect inducing an additional source of temporal variation in that same process. ${ }^{13}$

We assume that the representative agent's consumption and investment decisions are based on the maximization of Epstein-Zin-Weil recursive preferences. As formally shown in Appendix A, this implies the following equilibrium relationship between the intertemporal marginal rate of substitution, $M_{t}$, consumption, $C_{t}$, and the cumulated return on the aggregate wealth portfolio, $R_{t}$,

$$
\mathrm{d} \log M_{t}+\frac{\theta}{\psi} \mathrm{d} \log C_{t}+(1-\theta) \mathrm{d} \log R_{t}=-\rho \theta \mathrm{d} t
$$

where $\rho$ denotes the instantaneous subjective discount factor, $\psi$ equals the intertemporal elasticity of substitution, and the parameter $\theta$ is defined by

$$
\theta \equiv(1-\gamma)\left(1-\psi^{-1}\right)^{-1}
$$

where $\gamma$ refers to the coefficient of risk aversion. The expression in Equation (6) is naturally interpreted as the continuous-time version of the discrete-time equilibrium relationship derived in Epstein and Zin (1991). In the following, we will maintain the assumptions that $\gamma>1$ and $\psi>1$, which readily implies that $\theta<0 .{ }^{14}$ Consistent with the empirical regularities discussed in Section 1, these

\footnotetext{
$\overline{12}$ We also assume that $\mu_{q} \kappa_{q}>0.50 \varphi_{q}^{2}$, which ensures positivity of $q_{t}$, and that $\mu_{\sigma}$ is sufficiently large relative to $\kappa_{\sigma}$, so that negativity of $\sigma_{g, t}^{2}$ is highly unlikely and the subsequent approximations reasonable.

13 Empirical evidence in support of time-varying volatility-of-volatility in consumption growth has recently been presented in Bollerslev, Tauchen, and Zhou (2009). Alternatively, Drechsler and Yaron (2011) consider a discrete-time model with "jumps" in volatility (and expected growth rates), in part motivated by estimates reported in the option-pricing literature.

14 The assumption that $\gamma>1$ is generally agreed upon. Early estimates by, for example, Hall (1988) and Campbell and Mankiw (1989), put $\psi<1$, but these results have subsequently been called into question by Bansal and Yaron (2004) among many others, and we follow the more recent literature in assuming that $\psi>1$.
} 
specific parameter restrictions ensure, among other things, that asset prices fall on news of positive volatility shocks and that volatility carries a positive risk premium.

\subsection{INITIAL MODEL SOLUTION}

Let $\Psi_{t}=\Psi\left(\sigma_{g, t}^{2}, q_{t}, x_{t}\right)$ denote the price-dividend ratio, or equivalently the priceconsumption or the wealth-consumption ratio, of the asset that pay the consumption endowment $\left\{C_{t+s}\right\}_{s \in[0, \infty)}$. The equilibrium stochastic differential equation for $\log \left(\Psi_{t}\right)$ involves the reciprocal of $\Psi\left(z_{t}\right)$, which we approximate via $\Psi(z)^{-1} \approx \exp (-\overline{\log } \Psi)-\exp (-\overline{\log } \Psi)(\log \Psi(z)-\overline{\log } \Psi)=\kappa_{0}-\kappa_{1} \log \Psi(z)$, where $\kappa_{1}>0 .{ }^{15}$ Now, conjecturing a solution for $\log \left(\Psi_{t}\right)$ as an affine function of the three state variables, $\sigma_{g, t}^{2}, q_{t}$, and $x_{t}$,

$$
\log \left(\Psi_{t}\right)=A_{0}+A_{\sigma} \sigma_{g, t}^{2}+A_{q} q_{t}+A_{x} x_{t},
$$

and solving for the $A$ coefficients, it follows from Appendix B that

$$
\begin{gathered}
A_{0}=\frac{-\kappa_{0}-\rho+\left(1-\frac{1}{\psi}\right) \mu_{g}+A_{\sigma} \mu_{\sigma} \kappa_{\sigma}+A_{q} \mu_{q} \kappa_{q}+\frac{\theta A_{x}^{2} \sigma_{x}^{2}}{2}}{\kappa_{1}}, \\
A_{\sigma}=\frac{-\gamma\left(1-\frac{1}{\psi}\right)}{2\left(\kappa_{\sigma}+\kappa_{1}\right)}, \\
A_{q}=\frac{\kappa_{q}+\kappa_{1}-\sqrt{\left(\kappa_{q}+\kappa_{1}\right)^{2}-\theta^{2} \varphi_{q}^{2} A_{\sigma}^{2}}}{\theta \varphi_{q}^{2}}, \\
A_{x}=\frac{1-\frac{1}{\psi}}{\kappa_{x}+\kappa_{1}} .
\end{gathered}
$$

The restrictions that $\gamma>1$ and $\psi>1$ readily imply that the impact coefficient associated with both the volatility state variables are negative; that is, $A_{\sigma}<0$ and $A_{q}$ $<0 .{ }^{16}$ Or, put differently that the market falls on positive volatility "news." From

$\overline{15}$ This approximation plays a similar role to that of the standard Campbell-Shiller discrete-time approximation, and similar expressions have been used in a continuous-time setting by, for example, Campbell and Viceira (2002).

16 The solution for $A_{q}$ represents one of a pair of roots to a quadratic equation. However, it is the economically meaningful root as it implies that the premium disappears for $\varphi_{q} \rightarrow 0$, or when $q_{t}$ is constant, as would be required by the lack of arbitrage. 
these explicit solutions for the four coefficients, it is now possible to deduce the reduced-form expressions for other variables of interest.

In particular, as shown in Equation (B.13) in Appendix B, the equilibrium dynamics of the logarithmic cumulative return process is given by

$$
\begin{aligned}
\mathrm{d} \log R_{t}^{d}= & \left(\rho+\frac{\mu_{g}}{\psi}+\frac{\theta(\theta-1) A_{x}^{2} \sigma_{x}^{2}-\left((\theta-1) A_{x} \sigma_{x}+A_{x}^{d} \sigma_{x}+\phi_{\sigma x}\right)^{2}}{2}-\frac{\sigma_{d}^{2}}{2}\right. \\
& \left.+\frac{x_{t}}{\psi}-A_{q}^{d}\left(\kappa_{q}+\kappa_{1}^{d}\right) q_{t}-\left(A_{\sigma}^{d}\left(\kappa_{\sigma}+\kappa_{1}^{d}\right)+\frac{\phi_{\sigma}^{2}}{2}\right) \sigma_{g, t}^{2}\right) \mathrm{d} t \\
& +\phi_{\sigma} \sigma_{g, t} \mathrm{~d} W_{t}^{c}+\left(A_{x}^{d} \sigma_{x}+\phi_{\sigma x}\right) \mathrm{d} W_{t}^{x}+A_{\sigma}^{d} \sqrt{q_{t}} \mathrm{~d} W_{t}^{\sigma} \\
& +A_{q}^{d} \varphi_{q} \sqrt{q_{t}} \mathrm{~d} W_{t}^{q}+\sigma_{d} \mathrm{~d} W_{t}^{d} .
\end{aligned}
$$

The directional effects of increases in the endowment volatility, $\sigma_{g, t}^{2}$, on the local expected return are generally ambiguous. However, for sufficiently high levels of risk aversion $\gamma$ and intertemporal substitution $\psi$, endowment volatility positively affects the local expected return. Meanwhile, increases in the volatility-ofvolatility, $q_{t}$, unambiguously, increase the local expected return, reflecting the compensation for bearing volatility risk. On the other hand, diffusive-type innovations in the volatility and the volatility-of-volatility, $\mathrm{d} W_{t}^{\sigma}$ and $\mathrm{d} W_{t}^{q}$, both have a negative impact on the local returns, consistent with a leverage-type effect.

To further appreciate the implications of the model, it is instructive to consider the equity premium derived in Equation (B.12),

$$
\begin{aligned}
\pi_{r, t} \equiv-\frac{1}{\mathrm{~d} t} \frac{\mathrm{d}\left[R^{d}, M\right]_{t}}{R_{t}^{d} M_{t}} & =\gamma \phi_{\sigma} \sigma_{g, t}^{2}-(\theta-1)\left(A_{\sigma} A_{\sigma}^{d}+A_{q} A_{q}^{d} \varphi_{q}^{2}\right) q_{t} \\
& -(\theta-1)\left(A_{x} A_{x}^{d} \sigma_{x}+\phi_{\sigma x}\right) \sigma_{x} .
\end{aligned}
$$

The first term, $\gamma \sigma_{g, t}^{2}$, is akin to a classic risk-return trade-off relationship. It does not represent a volatility risk premium per se, however, but rather changing prices of consumption risk induced by the presence of stochastic volatility. Instead, the second term, $(1-\theta) \kappa_{1}^{2}\left(A_{\sigma} A_{\sigma}^{d}+A_{q} A_{q}^{d} \varphi_{q}^{2}\right) q_{t}$, has the interpretation of a true volatility risk premium, representing the confounding impact of the two diffusive-type innovations, $\mathrm{d} W_{t}^{\sigma}$ and $\mathrm{d} W_{t}^{q}$. The existence of this true volatility risk premium depends crucially on the dual assumptions of recursive utility, or $\theta \neq 1$, as volatility 
would not otherwise be a priced factor, and time-varying volatility-of-volatility, in the form of the $q_{t}$ process.

\subsection{CALIBRATION}

As a check on the initial model discussed above, we undertook a simple calibration to document that it is able to match the aggregate set of moments generally agreed upon in the macrofinance literature. The details are left to Appendix C.

The two main messages of the calibration are as follows. First, our initial model does indeed give a consistent equilibrium risk-based explanation of the dynamic cross-correlations between stock market return and volatility. This is evident by comparing the model-implied dynamic cross-dependencies displayed in the right-hand panel of Figure 3 to the observed dependencies seen in Figure 2. Evidently, the agreement is quite close. ${ }^{17}$ Second, as seen by contrasting the model-based autocorrelations in the left-hand panels of Figure 3 to the observed autocorrelations in Figure 1, the initial model is unable to account for the very slow decay in these that is so widely documented in the literature. ${ }^{18}$ This model failure can, of course, be traced directly to the presumed Markov specifications for the underlying dynamics.

The challenge, then, is to extend the underlying dynamics to incorporate longrange dependencies while at the same time preserving the equilibrium risk-based explanation for the dynamic leverage and volatility feedback effects seen in Figure 2. ${ }^{19}$ This extension entails some rather detailed technical analysis. Since we know from the stylized calibration in Appendix $C$ that the initial model is indeed capable of matching the generally agreed upon set of aggregate macrofinance moments, we subsequently simplify somewhat the analysis by assuming away the dynamic dependencies in the consumption endowment and dividend growth processes, while adapting the continuous-time setup and corresponding model solution to accommodate very flexible dynamic dependencies in the economic uncertainty, including long memory-type effects.

\footnotetext{
$\overline{17}$ Also, the instantaneous correlation between the changes in the VIX ${ }^{2}$ and the returns implied by the model calibrated in the appendix equals -0.418 and as such is entirely consistent with the corresponding numerically large empirical estimates reported in the extant literature.

18 The empirical and model-implied counterparts of the auto- and cross-correlations for the expected realized volatility, as formally defined below, closely parallel to those for the VIX ${ }^{2}$ depicted in Figures $1-3$. These additional results and graphs are available upon request.

19 To the best of our knowledge, with the exception of the distinctly different multifractal approach in Calvet and Fisher $(2007,2008)$, the extant equilibrium models all entail Markov dynamics and thereby cannot account for the long-range dependence evident in Figure 1.
} 


\subsection{GENERAL MODEL SOLUTION}

Numerous competing continuous-time stochastic volatility models have been proposed in the literature. We here build on the framework of Comte and Renault (1996) in assuming that $\sigma_{g, t}^{2}$ may be described by the general representation,

$$
\sigma_{g, t}^{2}=\sigma^{2}+\int_{-\infty}^{t} a(t-s) \sqrt{q_{s}} \mathrm{~d} W_{s}^{\sigma}
$$

By appropriate choice of the moving average weights $\{a(s)\}_{s \in[0, \infty)}$, this representation obviously includes the affine process in Equation (4) as a special case. Importantly, by suitable choice of the mapping $s \rightarrow a(s)$, the process for $\sigma_{g, t}^{2}$ may also exhibit various forms of long-range dependence. In particular, setting

$$
a(s)=\frac{\sigma}{\Gamma(1+\alpha)}\left(s^{\alpha}-k \mathrm{e}^{-k s} \int_{0}^{s} \mathrm{e}^{k u} u^{\alpha} \mathrm{d} u\right)
$$

results in the classic fractionally integrated process, where $\alpha$ denotes the longmemory parameter.

To complete the specification of the model and still allow for tractable closedform solutions, we will assume away the predictability in consumption growth in Equation (2), that is, $x_{t} \equiv 0$, while maintaining the identical law of motion for the volatility-of-volatility in Equation (5). The actual solution strategy, which is new and technically demanding, differs somewhat from that for the initial model. The full details are given in Appendix D; a precis follows.

In parallel to the solution for the short-memory model discussion above, we start by conjecturing a solution for the logarithmic price-consumption ratio now of the form

$$
\log \left(\Psi_{t}\right)=A_{0}+A_{q} q_{t}+\int_{-\infty}^{t} A(t-s) \sqrt{q_{s}} \mathrm{~d} W_{s}^{\sigma}
$$

where $A_{0}, A_{q}$, and $\{A(s)\}_{s \in[0, \infty)}$ are to be determined. Some care is needed because of subtleties related to possible arbitrage opportunities under long memory-type dependencies (Rogers, 1997). The strategy that we use relies on the fact that in the absence of arbitrage, the return on a traded security must follow a semimartingale. This allows us to split up the returns into a drift and a local martingale component. This decomposition is possible when $A(t)$ exists and is differentiable at zero. Substituting the conjectured solution into the pricing Equation (6), the following ordinary differential equation for $t>s$, 


$$
A^{\prime}(t-s)-\kappa_{1} A(t-s)=\frac{\gamma\left(1-\frac{1}{\psi}\right)}{2} a(t-s),
$$

and two regular equations,

$$
\begin{gathered}
\frac{\theta}{2} \varphi_{q}^{2} A_{q}^{2}-\left(\kappa_{q}+\kappa_{1}\right) A_{q}+\frac{\theta A(0)^{2}}{2}=0, \\
A_{0}=\frac{A_{q} \kappa_{q} \theta_{q}-\kappa_{0}+\left(1-\frac{1}{\psi}\right) \mu_{g}-\rho-\frac{\gamma}{2}\left(1-\frac{1}{\psi}\right) \sigma^{2}}{\kappa_{1}} .
\end{gathered}
$$

From the appendix, the solutions to this system of equations are

$$
\begin{gathered}
A(s)=-\int_{s}^{+\infty} \frac{\gamma\left(1-\frac{1}{\psi}\right)}{2} \mathrm{e}^{\kappa_{1}(t-\tau)} a(\tau) \mathrm{d} \tau \\
A_{q}=\frac{\kappa_{q}+\kappa_{1}-\sqrt{\left(\kappa_{q}+\kappa_{1}\right)^{2}-\theta^{2} \varphi_{q}^{2} A(0)^{2}}}{\theta \varphi_{q}^{2}}, \\
A_{0}=\frac{A_{q} \kappa_{q} \theta_{q}-\kappa_{0}+\left(1-\frac{1}{\psi}\right) \mu_{g}-\rho-\frac{\gamma}{2}\left(1-\frac{1}{\psi}\right) \sigma^{2}}{\kappa_{1}},
\end{gathered}
$$

which exists and is well-defined subject to a terminal condition ruling out explosive bubble solutions and other mild regularity conditions. As before, from this set of solutions, it is possible to deduce the reduced-form expressions for all other variables of interest.

In particular, in parallel to the expression for the returns in the short-memory model in Equation (9) above, it follows from Appendix D that the reduced-form expression for the returns in the general model may be expressed as,

$$
\mathrm{d} \log \left(R_{t}\right)=\mu_{R, t} \mathrm{~d} t+\sigma_{g, t} \mathrm{~d} W_{t}^{c}+A_{q} \varphi_{q} \sqrt{q_{t}} \mathrm{~d} W_{t}^{q}+A(0) \sqrt{q_{t}} \mathrm{~d} W_{t}^{\sigma},
$$

where the drift is defined by,

$$
\mu_{R, t}=\rho+\frac{\mu_{g}}{\psi}+\left[-\frac{1}{2}+\frac{\gamma}{2}\left(1-\frac{1}{\psi}\right)\right] \sigma_{g, t}^{2}-\left(\kappa_{q}+\kappa_{1}\right) A_{q} q_{t} .
$$

Similarly, from Equation (D.7) in Appendix C, the equilibrium equity premium takes the form, 


$$
\pi_{r, t}=\gamma \sigma_{t}^{2}+(1-\theta)\left[A_{q}^{2} \varphi_{q}^{2}+A(0)^{2}\right] q_{t}=\gamma \sigma_{t}^{2}+2\left(\frac{1}{\theta}-1\right)\left(\kappa_{q}+\kappa_{1}\right) A_{q} q_{t} .
$$

Under the previously discussed parameter restrictions $\gamma>1$ and $\psi>1$, implying that $\theta<0$, the equity premium remains positive. More generally, as long as $\gamma>\frac{1}{\psi}$, or $\theta<1$, it remains the case that stochastic volatility carries a positive risk premium. Note also that the instantaneous equity premium only depends on the $\{a(s)\}_{s \in[0, \infty)}$ weights and the possible long-run dependencies in the volatility through the cumulative impact determined by the integral solution for $A(0)$ in Equation (17).

\section{Dynamic Equilibrium Dependencies}

The equilibrium expressions discussed in the previous section characterize how the equity premium depends on the instantaneous volatility and how the instantaneous return responds to contemporaneous volatility innovations within the model. This section further details the model's implications in regard to the dynamic dependencies in the volatility and the volatility risk premium and how these volatility measures covary with leads and lags of the returns at different horizons. We will subsequently confront these theoretical predictions with the key empirical regularities discussed in Section 1.

\subsection{VIX AND THE VOLATILITY RISK PREMIUM}

One of the key features of the model is that the economic uncertainty reflected in $\sigma_{g, t}^{2}$ may exhibit long-range dependence, while the volatility of the uncertainty, $q_{t}$ is a short-memory process. This, in turn, has important implications for the serial correlation properties of the equivalent to the VIX volatility index implied by the model and the corresponding volatility risk premium and how these measures correlate with the returns.

To begin, consider the (forward) integrated variance, or quadratic variation, of the asset price $S_{t}$,

$$
\mathrm{IV}_{t, t+N} \equiv \int_{\tau=t}^{t+N} \mathrm{~d}[\log S, \log S]_{\tau}
$$

where the "square bracket" represents the standard quadratic variation process. From Equation (D.8) in Appendix D, the reduced-form expression for the integrated variance may be conveniently written as follows: 


$$
\mathrm{IV}_{t, t+N}=\int_{t}^{t+N} \sigma_{g, \tau}^{2} \mathrm{~d} \tau+\left(A_{q}^{2} \varphi_{q}^{2}+A(0)^{2}\right) \int_{t}^{t+N} q_{\tau} \mathrm{d} \tau
$$

The integrated variance is, of course, random and not observed until time $t+N$. The corresponding variance swap rate is defined as the time $t$ risk-neutralized expectation of $\mathrm{IV}_{t, t+N}$, say $E_{t}^{\mathcal{Q}}\left(\mathrm{IV}_{t, t+N}\right)$. This risk-neutral expectation may in theory be calculated in a completely model-free fashion from a cross section of option prices (see, e.g., Carr and Madan, 1998; Britten-Jones and Neuberger, 2000; Jiang and Tian, 2005). This way of calculating the variance swap rate directly mirrors the definition of the (squared) VIX volatility index for the S\&P 500,

$$
\mathrm{VIX}_{t}^{2} \equiv E_{t}^{\mathcal{Q}}\left(\mathrm{IV}_{t, t+N}\right)
$$

where the horizon $N$ is set to 1 month or 22 days. $^{20}$

This same risk-neutral expected variation may alternatively be calculated within the specific equilibrium model setting. In particular, it follows from Equation (D.9) in Appendix D that

$$
\mathrm{VIX}_{t}^{2}=\beta_{v x, 0}+\int_{-\infty}^{t} h_{v x}(t-s) \sqrt{q_{s}} \mathrm{~d} W_{s}^{\sigma}+\beta_{v x, q} q_{t},
$$

where the dependence on $N$ has been suppressed for notational convenience. The $\left\{h_{v x}(s)\right\}_{s \in[0, \infty)}$ weights depend on the $\{a(s)\}_{s \in[0, \infty)}$ moving average coefficients and importantly inherit any long-memory decay in those coefficients. As such, an eventual slow hyperbolic decay in the autocorrelations for VIX ${ }_{t}^{2}$ would therefore be entirely consistent with the implications from the general theoretical model; that is,

$$
\operatorname{Corr}\left(\mathrm{VIX}_{t}^{2}, \mathrm{VIX}_{t+s}^{2}\right)=c_{h} s^{b_{h}} \quad s>S,
$$

where $c_{h}>0$ and $b_{h}<0$ are constants and $S$ denotes a sufficiently long lag so that the short-run dependencies have dissipated.

Next, consider the variance risk premium, as formally defined by the difference between the risk-neutral and objective expectations of $\mathrm{IV}_{t, t+N}$,

$$
v p_{t} \equiv E_{t}^{\mathcal{Q}}\left(\mathrm{IV}_{t, t+N}\right)-E_{t}^{\mathcal{P}}\left(\operatorname{IV}_{t, t+N}\right) .
$$

Whereas $E_{t}^{\mathcal{Q}}\left(\mathrm{IV}_{t, t+N}\right)$ and $E_{t}^{\mathcal{P}}\left(\mathrm{IV}_{t, t+N}\right)$ both depend on the consumption growth volatility and the volatility-of-volatility of that process, the variance risk premium

$\overline{20}$ A more detailed description of the mechanical calculation of the VIX index is available in the white paper on the CBOE Web site; see also the discussion in Jiang and Tian (2007). 
is simply an affine function of the volatility-of-volatility or $q_{t}$. Specifically, from Equation (D.10) in Appendix D,

$$
v p_{t}=b_{v p, 0}+b_{v p, 1} q_{t},
$$

where $b_{v p, 0}>0$ and $b_{v p, 1}>0$, reflecting the positive premium for bearing volatility risk. Intuitively, for $\theta<1$, investors have a preference for early resolution of uncertainty, while $\psi>1$ implies that there is a negative link between the volatility and the $P / D$ ratio. Meanwhile, the SDF only depends indirectly on shocks to the volatility through $(\theta-1) R_{t}$. Thus, any asset that is positively correlated with volatility will be bearing a negative risk premium. As such, the premium for the variance risk exposure naturally increases if the uncertainty about volatility increases, that is, the volatility-of-volatility, as characterized by the $q_{t}$ process. Since the exposure of the variance swap to volatility shocks directly mirrors the exposure of the volatility, the variance premium that results from the covariance between the SDF and the variance swap therefore only depends on $q_{t}$.

Even though the variance risk premium will generally be positive, only if $q_{t}$ is time varying will the premium also be time varying. Moreover, from Equation (29) above, the $v p_{t}$ process simply inherits the dynamic dependencies in the $q_{t}$ process and should exhibit a relatively fast exponential decay in its autocorrelation structure. That is,

$$
\operatorname{Corr}\left(v p_{t}, v p_{t+s}\right)=c_{q} \mathrm{e}^{-\kappa_{q} s} \quad s>0,
$$

where $c_{q}>0$ denotes a positive constant.

\subsection{RETURN-VOLATILITY CORRELATIONS}

The equilibrium expressions for the variance swap rate and the premium discussed above also have some important and directly testable implications for the dynamic cross-correlations for the $\mathrm{VIX}_{t}^{2}$ and $v p_{t}$ with the returns. To help elucidate the economic mechanisms underlying these dependencies, it is instructive to first review the predictions under short-memory dynamics. We subsequently discuss the general case, explicitly allowing for long-memory dynamics. The cross-correlations between the variance premium and the returns are easier to calculate than those for the VIX, and we begin by considering these.

Let $r_{t} \equiv \mathrm{d} \log \left(R_{t}\right)$ denote the instantaneous return. We will refer to the crosscorrelations between the time $t$ premium $v p_{t}$ and the future returns, $r_{t+s}$ for $s>0$, as the forward correlations. The forward correlations represent the extent to which the premium is able to forecast the returns. The correlations between the premium $v p_{t}$ and the lagged returns, $r_{t+s}$ for $s<0$, on the other hand, represent 
the impact of movements in the past returns on the current variance premium. Given the well known near unpredictability of returns, we would expect the forward correlations to be positive, reflecting the premium for bearing volatility risk, but small and quickly declining to zero for longer interdaily return horizons. We would expect the lagged correlations to be negative, but increasing to zero for longer daily lags, consistent with the existence of a dynamic leverage-type effect. The formal theoretical predictions from the model confirm this intuition.

Specifically, from the results for the short-memory model derived in Appendix B, it follows that for $s>0$,

$$
\operatorname{Corr}\left(v p_{t}, r_{t+s}\right)=\beta_{R, q} \operatorname{Var}\left(q_{t}\right) K_{q} \mathrm{e}^{-\kappa_{q} s},
$$

where $\beta_{R, q}$ represents the sensitivity of the instantaneous returns to the $q_{t}$ process. Since $\beta_{R, q}>0$ and $K_{q}>0$, the forward correlations are all positive. Similarly, it follows from the appendix that the cross-correlations for $s<0$ satisfy,

$$
\operatorname{Corr}\left(v p_{t}, r_{t-s}\right)=\left(\beta_{R, q} \operatorname{Var}\left(q_{t}\right)+A_{q} \phi_{q}^{2} \mu_{q}\right) K_{q} \mathrm{e}^{-\kappa_{q} s} .
$$

Since the high-frequency returns are close to unpredictable, the value for $\beta_{R, q}$ is likely to be small. Hence, we would expect the second term involving $A_{q}<0$ to dominate the expression in parenthesis, and consequently, all the backward correlations to be negative. In summary, the model predicts,

$$
\operatorname{Corr}\left(v p_{t}, r_{t+s}\right)= \begin{cases}a_{-} \mathrm{e}^{-k_{q}|s|} & S<0, \\ a_{+} \mathrm{e}^{-k_{q} s} & S \geq 0,\end{cases}
$$

where $a_{-}<0$ and $a_{+}<0$. As discussed further below, this prediction does indeed adhere very closely with the pattern in the empirical correlations.

The dynamic cross-correlations between the VIX ${ }^{2}$ and the return are a bit more involved than those for the variance premium. Still, the basic intuition is essentially the same, except that the actual formulas now also depend on the volatility process $\sigma_{g, t}^{2}$ itself and its correlation with the returns. In particular, referring to Appendix B, the forward correlations for $s>0$ takes the form,

$$
\operatorname{Corr}\left(\operatorname{VIX}_{t}^{2}, r_{t+s}\right)=\beta_{R, \sigma} \operatorname{Var}\left(\sigma_{g, t}^{2}\right) K_{\sigma} \mathrm{e}^{-\kappa_{\sigma} s}+\beta_{R, q} \operatorname{Var}\left(q_{t}\right) K_{q} \mathrm{e}^{-\kappa_{q} s} .
$$

The sign of $\beta_{R, \sigma}$ will depend upon the preference parameters $\psi$ and $\gamma$. However, it may reasonably be expected to be positive, ${ }^{21}$ so that the forward cross-correlations will again be positive, with the decay toward zero ultimately determined by the dominant value of $\kappa_{\sigma}$ or $\kappa_{q}$. As for the premium, the backward correlations for $s<0$ are slightly more complicated, taking the form,

$\overline{21}$ The prototypical values $\psi=2.5$ and $\gamma=7.5$ used in our calibration imply that $\beta_{R, \sigma}=12.6$. 


$$
\begin{aligned}
\operatorname{Corr}\left(\operatorname{VIX}_{t}^{2}, r_{t-s}\right)= & \left(\beta_{R, \sigma} \operatorname{Var}\left(\sigma_{g, t}^{2}\right)+A_{\sigma} \mu_{\sigma}\right) K_{\sigma} \mathrm{e}^{-\kappa_{\sigma} s}+\left(\beta_{R, q} \operatorname{Var}\left(q_{t}\right)\right. \\
& \left.+A_{q} \phi_{q}^{2} \mu_{q}\right) K_{q} \mathrm{e}^{-\kappa_{q} s} .
\end{aligned}
$$

As discussed above, given the difficulties in predicting returns, we would expect the $\beta_{R, \sigma}$ and $\beta_{R, q}$ terms to be relatively small and dominated by the terms involving the $A_{\sigma}<0$ and $A_{q}<0$ coefficients that determine the instantaneous response of the returns to volatility innovations. Consequently, the backward correlations are naturally expected to be all negative. In summary,

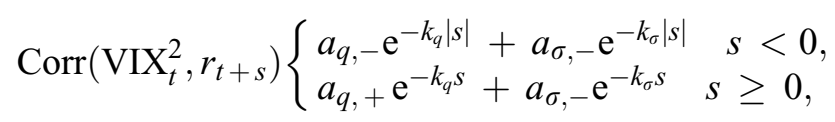

where $a_{q,-}, a_{\sigma,-}<0$ and $a_{q,+}, a_{\sigma,+}<0$. Again, these theoretical model predictions closely match what we see in the data.

The general model allowing for long memory in the economic uncertainty essentially gives rise to the same basic patterns and predictions. The formal derivations are somewhat more complicated; however, the actual values of the cross-correlations will ultimately depend upon the specific process for $\sigma_{g, t}^{2}$ and the corresponding moving average coefficients $\{a(s)\}_{s \in[0, \infty)}$. We briefly sketch the relevant tools and ideas required to evaluate the correlations.

The economics of the problem remain exactly the same. The main interactions between the return and the volatility are two-fold: one consists in the forward effect of volatility innovations on future expected returns and the other involves the feedback effect of lagged return innovations, or the diffusive part of the returns, on current volatility. To elucidate these separate effects within the general model setting, it is useful to define the auxiliary variable

$$
r_{t, s} \equiv\left\{\begin{array}{c}
\sigma_{g, t+s} \mathrm{~d} W_{t+s}^{c}+A_{q} \varphi_{q} \sqrt{q_{t+s}} \mathrm{~d} W_{t+s}^{q}+A(0) \sqrt{q_{t+s}} \mathrm{~d} W_{t+s}^{\sigma} \quad s<0, \\
\mu_{R, t+s} \quad s \geq 0,
\end{array}\right.
$$

which equals the local diffusive part of the equilibrium return process for $s<0$ and the local mean of the equilibrium return process for $s \geq 0$, respectively. As such, the basic shape of the cross-covariances between the variance risk premium $v p_{t}$ and $r_{t, s}$ directly mirrors that of the cross-covariances with the returns, $r_{t+s}$. In particular, it follows directly from the expression for $v p_{t}$ in Equation (29) that the forward correlations with $r_{t, s}$ must be proportional to the autocovariances of the $q_{t}$ process. That is, for $s>0$,

$$
\operatorname{Cov}\left(v p_{t}, r_{t, s}\right)=K_{r} \mathrm{e}^{-\kappa_{q} s},
$$

where $K_{r}>0$ denotes a positive constant of proportionality. To derive the backward correlations, write $q_{t}$ in integral form, 


$$
q_{t}=\varphi_{q} \int_{u=-\infty}^{t} \mathrm{e}^{\kappa_{q}(u-t)} \sqrt{q_{u}} \mathrm{~d} W_{u}^{q}
$$

From this expression, it follows that for $s<0$,

$$
\begin{aligned}
\operatorname{Cov}\left(v p_{t}, r_{t, s}\right) & =\mathrm{e}^{-\kappa_{q}|s|} E\left(b_{v p, 1} \varphi_{q} \sqrt{q_{t+s}} \mathrm{~d} W_{t+s}^{q}, A_{q} \varphi_{q} \sqrt{q_{t+s}} \mathrm{~d} W_{t+s}^{q}\right) \\
& =A_{q} b_{v p, 1} \varphi_{q}^{2} \mu_{q} \mathrm{e}^{-\kappa_{q}|s|},
\end{aligned}
$$

so that all the backward autocovariances are again negative and decay at an exponential rate, provided that $A_{q}<0$ as again implied by $\gamma>1$ and $\psi>1$. These dynamic patterns in the cross-covariances for $r_{t, s}$ directly translate into the crosscorrelations for $r_{t, s}$, and in turn the cross-correlations for the returns $r_{t+s}$, mirroring the implications from the short-memory model summarized in Equation (31) above.

The theoretical predictions for the dynamic cross-correlations between the VIX and the returns within the general model setting are not quite as clear-cut as those for the variance premium. The $q_{t}$ process determining the variance risk premium essentially gets confounded with the classical consumption risk premium, and there are also potential side effects from long-range dependence. However, the underlying economic mechanisms remain the same as for the short-memory model, resulting in a similar pattern of mixed negative backward correlations and positive forward correlations.

\section{Empirical Results}

The equilibrium framework developed above completely characterizes the dynamic dependencies in the returns and the volatility. Of course, the specific solution of the model will invariably depend upon the choice of preference parameters and the values of the parameters for the underlying consumption growth rate and volatility dynamics. Meanwhile, the model is obviously somewhat stylized, and direct estimation based on actual consumption data would be challenging at best. Instead, we next illustrate the model's qualitative implications in regard to the autocorrelations and cross-correlations derived in the previous section and, in particular, how well the basic patterns implied by the model match those of the actual data depicted in Figures 1 and 2 . We begin with a discussion of the data and pertinent summary statistics underlying the figures.

\subsection{DATA DESCRIPTION}

Our tick-by-tick data for the S\&P 500 futures contract were obtained from Tick Data Inc. To alleviate the impact of market microstructure "noise" in the 
calculation of the cross-correlations, and the realized volatility measures discussed further below, we follow the dominant approach in the literature and convert the tick-by-tick prices to equally spaced 5 -min observations. ${ }^{22}$ With seventyseven 5-min intervals per trading day and one overnight return, this leaves us with a total of seventy-eight "high-frequency" return observations per day. Standard summary statistics for the corresponding daily returns over the January 21990 through October 312007 sample period and the 5-min returns over the shorter September 232003 to August 312007 sample are reported in the first column in Table I.

The autocorrelations for the VIX in the top panel in Figure 1 are based on daily data from January 21990 through October 31 2007. These data are freely available from the CBOE. ${ }^{23}$ From the summary statistics reported in Table I, the average value of the VIX ${ }^{2}$ over the sample equals 32.81 , or 16.41 in standard deviation form, but it varies quite considerably over the sample, as indicated by the standard deviation of 23.70. This variation is quite persistent, however, as manifest by the slow decay in the aforementioned autocorrelations depicted in Figure 1. This strong persistence is also immediately evident from the actual time series plot of the data in Figure S1 of the supplementary material.

The tick-by-tick data for the VIX used in the construction of the crosscorrelations shown in the top panel in Figure 2 were again obtained from Tick Data Inc. High-frequency data for the VIX have only been available since the introduction of the "new" model-free VIX index on September 22 2003. The relevant summary statistics for the VIX ${ }^{2}$ over the shorter September 222003 to August 2007 high-frequency sample, reported in the bottom part of Table I, are broadly consistent with those over the longer daily sample. Of course, the kurtosis is substantially higher when the data are sampled at the 5-min frequency.

The integrated variance $\mathrm{IV}_{t, t+N}$ defined within the theoretical model is not directly observable. However, it may be consistently estimated in a completely model-free manner by the corresponding realized variation based on an increasing number of high-frequency squared returns over the fixed time interval $[t, t+N]$ (see, e.g., Andersen, Bollerslev, and Diebold, 2009). As previously noted, to guard against the adverse impact of market microstructure effects when sampling too

\footnotetext{
$\overline{22}$ The specific choice of a 5-min sampling frequency strike a reasonable balance between confounding market microstructure effects when sampling too frequently and the loss of important information concerning fundamental price movements when sampling more coarsely; see, for example, the discussion and references in Andersen et al. (2007), where the same futures data and 5-min sampling frequency have been used from a different perspective.

23 The VIX index is reported in annualized units by the CBOE. We convert the series to monthly units using the transformation $\mathrm{VIX}_{t}^{2}=30 / 365 \mathrm{VIX}_{\mathrm{CBOE}, t}^{2}$.
} 
frequently, we follow the common approach in the literature and rely on equally spaced, approximately serially uncorrelated, 5-min returns. ${ }^{24}$ With seventy-seven 5 -min intervals per trading day and the overnight return, the $\mathrm{N}$-day ahead realized variation is then simply given by,

$$
\mathrm{RV}_{t, t+N}=\sum_{i=1}^{78 N}\left(\log S_{t+i / 78}-\log S_{t+(i-1) / 78}\right)^{2}
$$

With the notable exception of a lower mean, reflecting a systematic premium for bearing volatility risk, the summary statistics for the 1 -month realized volatility measures $\mathrm{RV}_{t, t+22}$ reported in Table I are generally close to those for the VIX. The corresponding time series plots in Figures S1 and S2 also reveal the same general evolution in the two series.

The variance risk premium is formally defined as the difference between the objective and risk-neutralized expectations of the forward integrated variance. While the riskneutral expectation and the actually observed values of $\mathrm{IV}_{t, t+N}$ may both be estimated in a completely model-free fashion by the VIX and the realized volatilities, respectively, the calculation of the objective expectation $E_{t}\left(\mathrm{IV}_{t, t+N}\right)$ necessitates some mild auxiliary modeling assumptions. Motivated by the results in Andersen et al. (2003) that simple autoregressive-type models estimated directly for the realized volatility typically perform on par with, and often better, than specific parametric modeling approaches designed to forecast the integrated volatility, ${ }^{25}$ we will here rely on the Heterogeneous Autoregressive model of the Realized Volatility (HAR-RV) structure first proposed by Corsi (2009), and subsequently used by Andersen, Bollerslev, and Diebold (2007) among many others, in approximating the objective expectation. Specifically, define the 1-day ahead expectation by the linear projection of the realized volatility on the lagged daily, weekly, and monthly realized volatilities,

$$
E_{t}\left(\mathrm{RV}_{t, t+1}\right)=\beta_{r \mathrm{v}, 0}+\beta_{r \mathrm{v}, 1} \mathrm{RV}_{t-1, t}+\beta_{r \mathrm{v}, 2} \mathrm{RV}_{t-5, t}+\beta_{r \mathrm{v}, 2} \mathrm{RV}_{t-22, t} .
$$

\footnotetext{
$\overline{24}$ The first- and second-order autocorrelations and their robust standard errors (in parentheses) equal $-0.034(0.005)$ and $-0.008(0.004)$, respectively. Although statistically significant at conventional levels, these are obviously very small numerically and immaterial in terms of the resulting realized volatilities. Recent studies (e.g., Zhang, Mykland, and Aït-Sahalia, 2005; Barndorff-Nielsen et al., 2008) have proposed more efficient ways in which to annihilated the impact of the market microstructure "noise," permitting even finer sampling. However, the simple-to-implement realized variance estimator used here remains the dominant approach in practice and importantly allows for easy verification and replication of the results.

25 Andersen, Bollerslev, and Meddahi (2004) have formally shown that for the stochastic volatility models most commonly employed in the literature, the loss in efficiency from the use of reduced-form autoregressive models for the realized volatility is typically small; see also Sizova (2009).
} 
The 1-month expectation $E_{t}\left(\mathrm{IV}_{t, t+22}\right)=E_{t}\left(\mathrm{RV}_{t, t+22}\right)$ is then simply obtained by iterating the projection forward. ${ }^{26}$

The summary statistics for the resulting variance risk premium $\widehat{v} p_{t}=\mathrm{VIX}_{t}^{2}-\widehat{E} \mathrm{RV}_{t, t+22}$, reported in the last column in Table I, not only confirm the positive expected return for selling volatility but also show that the magnitude of the premium varies substantially over time. At the same time, the plots in Figures $\mathrm{S} 1$ and S2 indicate much less persistent dependencies in the premium than for the VIX and the realized volatilities. We next turn to our discussion of the theoretical model's ability to match this important feature along with the other key dynamic dependencies observed in the high-frequency data.

\subsection{MODEL-IMPLIED AUTO- AND CROSS-CORRELATIONS}

A full characterization of the model-implied autocorrelations for the integrated volatility would require that all the moving average weights $\{a(s)\}_{s \in[0, \infty)}$ in Equation (11) be completely specified. Importantly, however, as discussed in Section 3.1, any long-run dependencies in these coefficients directly translate to similar long-run dependencies in the moving average weights $\left\{h_{v x}(s)\right\}_{s \in[0, \infty)}$ that describe the equilibrium process for the VIX ${ }_{t}^{2}$ in Equation (26). The top panel in Figure 4 shows the best fitting model-implied autocorrelations from estimating the slowly hyperbolic decaying autocorrelation structure in Equation (27) to the actual daily sample autocorrelations for the VIX starting at a lag length of $S=22 .{ }^{27}$ The figure also shows the conventional $95 \%$ confidence intervals for the sample autocorrelations. The model-implied autocorrelations do a remarkable job at describing the long-run dependencies inherent in the VIX, always falling well inside the confidence bands. Of course, the fit does not match at all well if extrapolated to the 1- to 22-day interval, which is to be expected.

One of the key predictions of the theoretical model is that the equilibrium volatility risk premium is an affine function of the volatility-of-volatility and thereby is short memory, despite the fact that the integrated volatility and its risk-neutral expectation may both display long-memory dependencies. Intuitively, as discussed above, everything except for the volatility-of-volatility gets risk neutralized out in Equation (29). This, of course, is consistent with the shape of the autocorrelation function displayed in Figure 1, which in sharp contrast to the one for the VIX dies out relatively quickly. As a more formal verification of this distinct implication from the model, we fitted the functional form in Equation (30) to the actual sample

\footnotetext{
$\overline{26}$ The actual estimates for the $\beta$ s are directly in line with the results reported in the extant literature and available upon request.

27 The $R^{2}$ from estimating the functional relationship is an impressive 0.993 , although this value should be carefully interpreted because of the strong serial correlation in the residuals from the fit.
} 
autocorrelations for the variance risk premium. The fit shown in the bottom panel of Figure 4 is again excellent, and the model-implied autocorrelations easily fall within the $95 \%$ confidence intervals over the entire 1 - to 90 -day range. ${ }^{28}$

The observed volatility feedback and leverage effects evident in the dynamic cross-correlations in Figure 2 arguably present the more challenging and difficult to explain empirical dependencies. Consider first the cross-correlations for the variance risk premium. The negative backward correlations start out at a slightly larger absolute value than the forward correlations and both decay toward zero at what appears to be an exponential rate. This apparent pattern in the cross-correlations between the premium and leads and lags of the returns is entirely consistent with the model-implied correlations summarized in Equation (31). The bottom panel in Figure 5 shows the resulting fit along with the 95\% confidence intervals for the sample cross-correlations. ${ }^{29}$ The theoretical model obviously delivers highly accurate predictions for the actually observed dynamic dependencies between the returns and the variance risk premium.

The theoretical predictions for the VIX-return cross-correlations are not quite as clear-cut as those for the premium. As discussed in Section 3.2 above, the volatility risk premium in effect gets confounded with the classical consumption risk premium, and within the general theoretical model setting, there may also be potential side effects from long-range dependence. In parallel to the model-implied autocorrelations for the VIX, a complete characterization of these separate effects would require that the underlying fundamental consumption growth rate volatility process $\sigma_{g, t}^{2}$ and the corresponding moving average weights $\{a(s)\}_{s \in[0, \infty)}$ be fully specified. Short of such a specification, the basic pattern and decay in the cross-correlations may naturally be expected to adhere to the functional form in Equation (32). The top panel in Figure 5 shows the resulting fit to the sample cross-correlations.

Comparing the observed backward correlations for the VIX in the left part of Figure 2 to those for the variance premium in the bottom panel, the dynamic leverage effect is clearly more prolonged for the VIX. This slower decay is very well described by the mixed exponential functions shown in Figure 5. At the same time, the differences between the forward correlations for the VIX and the premium, and in turn the impact on future returns attributable to the classical consumption risk premium and mean-variance trade-off, appear less pronounced. In fact, the

\footnotetext{
$\overline{28}$ The fitted $R^{2}$ equals 0.934 .

29 The fitted $R^{2}$ s for the backward and forward correlations equal 0.907 and 0.678 , respectively. Of course, some of the sample cross-correlations used in the fit are not statistically different from zero.
} 
relatively fast decay rates in the empirically observed forward correlations are well described by a single exponential function for both the premium and the VIX. ${ }^{30}$

Summing up the empirical results, the qualitative implications from the new theoretical model do an admirable job in terms of matching the key dynamic dependencies in the aggregate market returns and volatilities. The previously documented autocorrelations for the volatility and volatility risk premium and the puzzling high frequency-based cross-correlation patterns in Figures 1 and 2 may all be explained by the model, with the model predictions well within conventional statistical confidence intervals.

\section{Conclusion}

Aggregate stock market volatility exhibits long memory-type dependencies, while the variance risk premium, defined as the difference between the objective and the risk-neutral expectations of the forward variance, shows much less persistence. Consistent with the well-documented leverage and volatility feedback effects, there is also a distinct and prolonged asymmetry in the relationship between volatility and past and future returns. We provide the first self-contained equilibrium-based explanation for all these empirical facts. The return on the aggregate market defined within the new model depends not only on the prospects of future economic growth but also on the current uncertainty about the future economic conditions, thereby explaining the presence of a separate premium for bearing variance risk through a preference for early resolution of uncertainty.

Our explanation of the empirical facts is entirely risk based and depends critically on the temporal variation in the variance risk premium defined within the model. The wedge between the objective and the risk-neutral expectations of the forward variance may alternatively be interpreted as a proxy for the aggregate degree of risk aversion in the market and any temporal variation in the empirically observed variance risk premium, thus indicative of changes in the way in which systematic risk is valued (see, e.g., Aït-Sahalia and Lo, 2000; Bollerslev, Gibson, and Zhou, 2011; Gordon and St-Amour, 2004; Vanden, 2005). Although it might be difficult to contemplate systematic changes in the level of risk aversion at the frequencies emphasized here, time-varying volatility risk and time-varying attitudes toward risk likely both play a role in explaining the temporal variation in expected returns and risk premia (e.g., Bekaert, Engstrom, and Xing, 2009). It would be interesting to extend the new framework and model developed here to explicitly allow for changes in the underlying preference parameters and risk attitudes to help delineate these effects.

$\overline{30}$ The fitted $R^{2}$ s for a double exponential for the VIX backward correlations and a single exponential for the VIX forward correlations equal 0.941 and 0.709 , respectively. 
This may be especially important in understanding the dynamic dependencies and high-frequency feedback effects observed during the recent financial crises.

\section{Supplementary Material}

Supplementary data are available at Review of finance online.

\section{Appendix A:Continuous-Time Equilibrium and SDF}

The generalized preferences that we use are a continuous-time version of the Epstein-Zin-Weil discrete-time utility:

$$
\tilde{V}_{t}^{1-\frac{1}{\psi}}=\left(1-\mathrm{e}^{-\rho h}\right) C_{t}^{1-\frac{1}{\psi}}+\mathrm{e}^{-\rho h}\left[E_{t} \tilde{V}_{t+h}^{1-\gamma}\right]^{1-\frac{1}{\psi}} .
$$

Assuming that $\tilde{V}_{t}^{1-\gamma}$ is a semimartingale, we approximate its conditional expectation over a short time interval $h$ by the linear function:

$$
E_{t} \tilde{V}_{t+h}^{1-\gamma} \approx D\left(\tilde{V}_{t}^{1-\gamma}\right) h+\tilde{V}_{t}^{1-\gamma},
$$

where $D($.$) denotes the drift of the argument. Plugging the conditional expectation$ in Equation (A.2) into the definition (A.1), and taking limits around $h=0$, the drift for the utility may be expressed as a function of consumption and utility:

$$
D\left(\tilde{V}_{t}^{1-\gamma}\right)=\theta \rho\left[1-\frac{C_{t}^{1-\frac{1}{\psi}}}{\tilde{V}_{t}^{1-\frac{1}{\psi}}}\right] \tilde{V}_{t}^{1-\gamma} .
$$

The original utility $\tilde{V}_{t}$ in Equation (A.1) can be replaced by any ordinally equivalent utility $V_{t}=\varphi\left(\tilde{V}_{t}\right)$, where the transformation $\varphi($.$) is strictly increasing. Fol-$ lowing Duffie and Epstein (1992b), we apply the transformation $\varphi($.$) that is linear in$ $\tilde{V}_{t}^{1-\gamma}$ :

$$
V_{t}=\frac{1}{1-\gamma} \tilde{V}_{t}^{1-\gamma}
$$

Given this choice of $\varphi($.$) , the preferences may be simply defined through the$ recursive condition:

$$
D V_{t}+f\left(C_{t}, V_{t}\right)=0,
$$

where the normalized drift equals 


$$
f(c, v)=\frac{\rho}{1-\frac{1}{\psi}} \frac{c^{1-\frac{1}{\psi}}-[(1-\gamma) v]^{1 / \theta}}{[(1-\gamma) v]^{1 / \theta-1}} .
$$

This in effect constitutes the formal definition of the Epstein-Zin-Weil preferences in continuous time; see also Duffie and Epstein (1992a).

\section{A.1 SDF IN CONTINUOUS TIME}

In this section, we derive the exact formula for the SDF under the Epstein-ZinWeil preferences in Equation (A.4) as a function of the return on the consumption asset and the consumption growth rate.

For notational convenience, denote the logarithmic welfare-consumption ratio:

$$
v c_{t} \equiv \log \frac{\tilde{V}_{t}}{C_{t}}=\frac{1}{1-\gamma} \log \left([1-\gamma] V_{t}\right)-\log C_{t} .
$$

The normalized drift in Equation (A.4) may then alternatively be represented as follows:

$$
f\left(C_{t}, V_{t}\right)=\rho \theta V_{t}\left(e^{-\left(1-\frac{1}{\psi}\right) v c_{t}}-1\right)
$$

Duffie and Epstein (1992a) have previously derived the SDF for recursive preferences with an arbitrary normalized $\operatorname{drift} f\left(C_{t}, V_{t}\right)$ :

$$
M_{t}=\exp \int_{0}^{t} f_{v}\left(C_{s}, V_{s}\right) \mathrm{d} s f_{c}\left(C_{t}, V_{t}\right) .
$$

The dynamics for the SDF thus follows from the dynamics of the two partial derivatives,

$$
f_{c} \equiv \frac{\partial f}{\partial C_{t}}=(1-\gamma) \rho C_{t}^{-\gamma} \mathrm{e}^{-\log (1-\gamma)+\left(\frac{1}{\psi}-\gamma\right) \nu c_{t}},
$$

and

$$
f_{v} \equiv \frac{\partial f}{\partial V_{t}}=\theta \rho\left(\mathrm{e}^{-\left(1-\frac{1}{\psi}\right) v c_{t}}-1\right)\left(1-\frac{1}{\theta}\right)-\rho .
$$

Now, note that

$$
V_{t}^{1 / \theta} M_{t} C_{t}^{1 / \psi}=\rho(1-\gamma)^{1-1 / \theta} V_{t} \mathrm{e}^{t}{ }_{0}^{t} f_{\mathrm{v}} \mathrm{d} s .
$$


Extracting the drift of the process on the left-hand side of the above equation, taking into account that the drift of the $V_{t}$ process equals $-f\left(C_{t}, V_{t}\right)$, and rearranging the terms, we obtain the following relation:

$$
D\left[\frac{\mathrm{e}^{\frac{\log (1-\gamma)}{\theta}}}{\rho}\left(\frac{V_{t}^{\frac{1}{1-\gamma}}}{C_{t}}\right)^{1-\frac{1}{\psi}} M_{t} C_{t}\right]=-M_{t} C_{t} .
$$

In other words,

$$
\frac{\mathrm{e}^{\frac{\log (1-\gamma)}{\theta}}}{\rho}\left(\frac{V_{t}^{\frac{1}{1-\gamma}}}{C_{t}}\right)^{1-\frac{1}{\psi}}
$$

satisfies the Euler condition for the price-dividend ratio of the consumption asset. Together with appropriate terminal conditions, this implies that this expression must be equal to the price-dividend ratio. In logarithm terms, there is a one-toone correspondence between the price-dividend ratio of the consumption asset and the welfare-consumption ratio:

$$
\log \Psi_{t}=-\log \rho+\left(1-\frac{1}{\psi}\right) v c_{t}
$$

The formula for the price-dividend ratio implies that the total return satisfies:

$$
\frac{\mathrm{d} R_{t}}{R_{t}} \equiv \frac{\mathrm{d} S_{t}+C_{t} \mathrm{~d} t}{S_{t}}=\frac{\mathrm{d}\left[\Psi_{t} C_{t}\right]}{\Psi_{t} C_{t}}+\frac{\mathrm{d} t}{\Psi_{t}}
$$

Combining the definitions of the return in Equation (A.8), the SDF in Equation (A.6), and the price-dividend ratio in Equation (A.7), we obtain the following relation:

$$
\begin{aligned}
& \mathrm{d} \log M_{t}+(1-\theta) \mathrm{d} \log R_{t}+\frac{\theta}{\psi} \mathrm{d} \log C_{t}=\left(f_{v}\left(C_{t}, V\right) \mathrm{d} t+\mathrm{d} \log f_{c}\left(C_{t}, V_{t}\right)\right) \\
& +(1-\theta)\left(\mathrm{d} \log C_{t}+\mathrm{d} \log \Psi_{t}+\frac{\mathrm{d} t}{\Psi_{t}}\right)+\frac{\theta}{\psi} \mathrm{d} \log C_{t}=-\rho \theta \mathrm{d} t
\end{aligned}
$$

where the last equality follows from two identities:

$$
f_{v}\left(C_{t}, V_{t}\right)+(1-\theta) \frac{1}{\Psi_{t}}=\rho \theta
$$




$$
\mathrm{d} \log f_{c}\left(C_{t}, V_{t}\right)+(1-\theta)\left(\mathrm{d} \log C_{t}+\mathrm{d} \log \Psi_{t}\right)+\frac{\theta}{\psi} \mathrm{d} \log C_{t}=0
$$

The expression for the SDF in Equation (A.9) as a function of the return on aggregate consumption and consumption growth may naturally be seen as the continuous-time version of the similar discrete-time relationship in Bansal and Yaron (2004). We next proceed to study the asset pricing implications of the model and this SDF, including expressions for the risk-free rate, the return on the consumption asset, and the variance risk premium.

\section{Appendix B:Model Solution under Short-Memory Dynamics}

\section{B.1 PRICING OF THE CONSUMPTION AND DIVIDEND ASSETS}

Suppose, that the dynamics of the consumption growth $g_{t} \equiv \frac{\mathrm{d} C_{t}}{C_{t}}$ is determined by the following system of equations:

$$
\begin{aligned}
\frac{\mathrm{d} C_{t}}{C_{t}} & =\left(\mu_{g}+x_{t}\right) \mathrm{d} t+\sigma_{g, t} \mathrm{~d} W_{t}^{c}, \\
\mathrm{~d} x_{t} & =-\kappa_{x} x_{t} \mathrm{~d} t+\sigma_{x} \mathrm{~d} W_{t}^{x}, \\
\mathrm{~d} \sigma_{g, t}^{2} & =\kappa_{\sigma}\left(\mu_{\sigma}-\sigma_{g, t}^{2}\right) \mathrm{d} t+\sqrt{q_{t}} \mathrm{~d} W_{t}^{\sigma}, \\
\mathrm{d} q_{t} & =\kappa_{q}\left(\mu_{q}-q_{t}\right) \mathrm{d} t+\varphi_{q} \sqrt{q_{t}} \mathrm{~d} W_{t}^{q},
\end{aligned}
$$

where all the shocks are uncorrelated. Under the risk-neutral measure, the asset return is a martingale with respect to information at time $t$, that is,

$$
D\left(M_{t} R_{t}\right)=0 .
$$

It follows from the formula for the SDF in Equation (A.9) and the definition of the return in Equation (A.8) that

$$
\mathrm{d} \log \left(M_{t} R_{t}\right)=\theta\left(\mathrm{d} \log \Psi_{t}+\mathrm{d} \log C_{t}+\frac{\mathrm{d} t}{\Psi_{t}}\right)-\frac{\theta}{\psi} \mathrm{d} \log C_{t}-\rho \theta \mathrm{d} t .
$$

Substituting the price-dividend ratio $\Psi_{t} \equiv \Psi_{t}\left(X_{t}\right)$ into the above condition for the drift $D\left(M_{t} R_{t}\right)$ yields the following pricing relation: 


$$
\begin{gathered}
\theta D \log \Psi\left(X_{t}\right)+\frac{\theta}{\Psi\left(X_{t}\right)}+(1-\gamma)\left(\mu_{g}+x_{t}-\frac{\sigma_{g, t}^{2}}{2}\right)-\rho \theta \\
+\frac{\theta^{2}}{2} \frac{\mathrm{d}[\log \Psi(X), \log \Psi(X)]_{t}}{\mathrm{~d} t}+\frac{1}{2}(1-\gamma)^{2} \sigma_{g, t}^{2}=0,
\end{gathered}
$$

where $D \log \Psi\left(X_{t}\right)$ denotes the drift of $\log \Psi\left(X_{t}\right)$ and $[\log \Psi(X), \log \Psi(X)]_{t}$ refers to the quadratic variation, whose increment characterizes the variance of shocks to log $\Psi\left(X_{t}\right)$. The pricing relation in Equation (B.5) may now be solved using the firstorder approximations similar to Campbell and Viceira (2002),

$$
\begin{aligned}
\frac{1}{\Psi\left(X_{t}\right)} & =\exp \left(-\log \Psi\left(X_{t}\right)\right) \\
& \approx \exp (-\log \Psi)-\exp (-\log \Psi)\left(\log \Psi\left(X_{t}\right)-\log \Psi\right) .
\end{aligned}
$$

In particular, under this linearization, it is natural to conjecture that log pricedividend ratio is linear in the states:

$$
\log \Psi\left(X_{t}\right)=A_{0}+A_{x} x_{t}+A_{\sigma} \sigma_{g, t}^{2}+A_{q} q_{t},
$$

and therefore,

$$
\frac{1}{\Psi\left(X_{t}\right)} \approx-\kappa_{0}-\kappa_{1}\left(A_{0}+A_{x} x_{t}+A_{\sigma} \sigma_{g, t}^{2}+A_{q} q_{t}\right) .
$$

Substituting the conjectured solution for $\Psi\left(X_{t}\right)$ in Equation (B.6) and its inverse value in Equation (B.7) into the pricing condition (B.5), we find the coefficients:

$$
\begin{aligned}
& A_{0}=\frac{A_{\sigma} \kappa_{\sigma} \mu_{\sigma}+A_{q} \kappa_{q} \mu_{q}-\kappa_{0}+(1-1 / \psi) \mu_{g}-\rho+\frac{\theta A_{x} \sigma_{x}^{2}}{2}}{\kappa_{1}}, \\
& A_{x}=\frac{1-\frac{1}{\psi}}{\kappa_{x}+\kappa_{1}}, \\
& A_{\sigma}=-\frac{\gamma}{2} \frac{1-\frac{1}{\psi}}{\kappa_{\sigma}+\kappa_{1}}, \\
& A_{q}=\frac{\kappa_{q}+\kappa_{1}-\sqrt{\left(\kappa_{q}+\kappa_{1}\right)^{2}-\theta^{2} A_{\sigma}^{2} \varphi_{q}^{2}}}{\theta \varphi_{q}^{2}}
\end{aligned}
$$


where the value of $A_{q}$ is the root of a quadratic equation that is bounded away from $\infty$ as $\phi_{q}$ goes to zero. Note that similar to the discrete-time case, the loadings $A_{\sigma}$ and $A_{q}$ are negative for values of the intertemporal elasticity of substitution $\psi>1$.

Combining the dynamics of dividends $C_{t}$ and the dynamics of the price-dividend ratio, we obtain the dynamics for the total log return under the objective measure:

$$
\begin{aligned}
\mathrm{d} \log R_{t} & =\left(\frac{\mu_{g}+x_{t}}{\psi}+\rho-\frac{\theta A_{x}^{2} \sigma_{x}^{2}}{2}+\left(-\frac{1}{2}+\frac{\gamma}{2}\left(1-\frac{1}{\psi}\right)\right) \sigma_{g, t}^{2}\right. \\
& \left.-A_{q}\left(\kappa_{1}+\kappa_{q}\right) q_{t}\right) \mathrm{d} t+\sigma_{g, t} \mathrm{~d} W_{t}^{c}+A_{x} \sigma_{x} \mathrm{~d} W_{t}^{x}+A_{\sigma} \sqrt{q_{t}} \mathrm{~d} W_{t}^{\sigma} \\
& +A_{q} \varphi_{q} \sqrt{q_{t}} \mathrm{~d} W_{t}^{q} .
\end{aligned}
$$

The dynamics for the SDF follows from Equation (A.9):

$$
\begin{aligned}
\frac{\mathrm{d} M_{t}}{M_{t}}= & \left(\frac{\gamma}{2}\left(1+\frac{1}{\psi}\right) \sigma_{g, t}^{2}-\left(1-\frac{1}{\theta}\right)\left(\kappa_{q}+\kappa_{1}\right) A_{q} q_{t}-\frac{1}{\psi}\left(\mu_{g}+x_{t}\right)-\rho\right. \\
& \left.-\frac{(\theta-1) A_{x}^{2} \sigma_{x}^{2}}{2}\right) \mathrm{d} t-\gamma \sigma_{g, t} \mathrm{~d} W_{t}^{g}+(\theta-1)\left(A_{x} \sigma_{x} \mathrm{~d} W_{t}^{x}+A_{\sigma} \sqrt{q_{t}} \mathrm{~d} W_{t}^{\sigma}\right. \\
& \left.+A_{q} \varphi_{q} \sqrt{q_{t}} \mathrm{~d} W_{t}^{q}\right)
\end{aligned}
$$

The dynamics of SDF readily defines the risk-free rate, the equity premium, and the risk-neutral probability measure. For example, the risk-free rate is simply given by the drift of the SDF:

$$
\begin{aligned}
r_{f, t} \equiv-E_{t} \frac{\mathrm{d} M_{t}}{M_{t}}= & \frac{\mu_{g}+x_{t}}{\psi}+\rho+\frac{(\theta-1) A_{x}^{2} \sigma_{x}^{2}}{2}-\frac{\gamma}{2}\left(1+\frac{1}{\psi}\right) \sigma_{g, t}^{2} \\
& +\left(1-\frac{1}{\theta}\right)\left(\kappa_{q}+\kappa_{1}\right) A_{q} q_{t} .
\end{aligned}
$$

The diffusion part of the SDF in Equation (B.9) defines the transition from the processes under the objective measure to the risk-neutral measure:

$$
\begin{aligned}
\frac{\mathrm{d} C_{t}}{C_{t}} & =\left(\mu_{g}-\gamma \sigma_{g, t}^{2}\right) \mathrm{d} t+\sigma_{g, t} \mathrm{~d} \tilde{W}_{t}^{c}, \\
\mathrm{~d} x_{t} & =\left(-\kappa_{x} x_{t}+(\theta-1) A_{x} \sigma_{x}^{2}\right) \mathrm{d} t+\sigma_{x} \mathrm{~d} \tilde{W}_{t}^{x},
\end{aligned}
$$




$$
\begin{aligned}
\mathrm{d} \sigma_{g, t}^{2} & =\left(\kappa_{\sigma}\left(\mu_{\sigma}-\sigma_{g, t}^{2}\right)+(\theta-1) A_{\sigma} q_{t}\right) \mathrm{d} t+\sqrt{q_{t}} \mathrm{~d} \tilde{W}_{t}^{\sigma}, \\
\mathrm{d} q_{t} & =\left(\kappa_{q}\left(\mu_{q}-q_{t}\right)+(\theta-1) A_{q} \varphi_{q}^{2} q_{t}\right) \mathrm{d} t+\varphi_{q} \sqrt{q_{t}} \mathrm{~d} \tilde{W}_{t}^{q},
\end{aligned}
$$

where $\mathrm{d} \tilde{W}_{t}^{c}, \mathrm{~d} \tilde{W}_{t}^{x}, \mathrm{~d} \tilde{W}_{t}^{\sigma}$, and $\mathrm{d} \tilde{W}_{t}^{q}$ are all uncorrelated Wiener processes under the risk-neutral probability measure.

Consider an arbitrary asset with a general dynamics for dividends:

$$
\frac{\mathrm{d} D_{t}}{D_{t}}=\left(\mu_{d}+\phi_{x} x_{t}\right) \mathrm{d} t+\phi_{\sigma} \sigma_{g, t} \mathrm{~d} W_{t}^{c}+\phi_{\sigma, x} \mathrm{~d} W_{t}^{x}+\sigma_{d} \mathrm{~d} W_{t}^{d},
$$

where $\mathrm{d} W_{t}^{d}$ is an idiosyncratic shock, that is, independent of any process that drives consumption. A similar conjecture for the price-dividend ratio,

$$
\log \Psi^{d}\left(X_{t}\right)=A_{0}^{d}+A_{x}^{d} x_{t}+A_{\sigma}^{d} \sigma_{g, t}^{2}+A_{q}^{d} q_{t},
$$

yields a solution that satisfies no-arbitrage condition:

$$
\begin{gathered}
A_{x}^{d}=\frac{\phi_{x}-\frac{1}{\psi}}{\kappa_{x}+\kappa_{1}^{d}}, \\
A_{\sigma}^{d}=-\frac{\gamma}{2} \frac{2 \phi_{\sigma}-1-\frac{1}{\psi}}{\kappa_{\sigma}+\kappa_{1}^{d}}, \\
A_{q}^{d}=\frac{\frac{\kappa_{1}^{d}+\kappa_{q}}{\varphi_{q}}-\sqrt{\left(\frac{\kappa_{1}^{d}+\kappa_{q}}{\varphi_{q}}\right)^{2}-\left((\theta-1) A_{\sigma}+A_{\sigma}^{d}\right)^{2}-2(\theta-1) A_{q}\left(\kappa_{1}^{d}-\kappa_{1}\right)}}{\varphi_{q}} \\
-(\theta-1) A_{q},
\end{gathered}
$$

where $\kappa_{0}^{d}$ and $\kappa_{1}^{d}$ are coefficients from the linear approximation $\frac{1}{\Psi^{d}\left(X_{t}\right)} \approx-\kappa_{0}^{d}-\kappa_{1}^{d} \log \Psi^{d}\left(X_{t}\right)$.

The equity premium paid on this asset is obtained as the "covariance" between the total return and the SDF:

$$
\begin{aligned}
\pi_{r, t} \equiv \frac{1}{\mathrm{~d} t} \frac{\mathrm{d}\left[R^{d}, M\right]_{t}}{R_{t}^{d} M_{t}} & =\gamma \phi_{\sigma} \sigma_{g, t}^{2}-(\theta-1)\left(A_{x} A_{x}^{d} \sigma_{x}+\phi_{\sigma, x}\right) \sigma_{x} \\
& -(\theta-1)\left(A_{\sigma} A_{\sigma}^{d}+A_{q} A_{q}^{d} \varphi_{q}^{2}\right) q_{t} .
\end{aligned}
$$


The instantaneous return is a function of an increment in the price-dividend ratio and the paid dividends:

$$
\begin{aligned}
\mathrm{d} \log R_{t}^{d}= & \left(\rho+\frac{\mu_{g}+x_{t}}{\psi}+\frac{\theta(\theta-1) A_{x}^{2} \sigma_{x}^{2}-\left((\theta-1) A_{x} \sigma_{x}+A_{x}^{d} \sigma_{x}+\phi_{\sigma, x}\right)^{2}}{2}\right. \\
& \left.-\frac{\sigma_{d}^{2}}{2}-A_{q}^{d}\left(\kappa_{q}+\kappa_{1}^{d}\right) q_{t}-\left(A_{\sigma}^{d}\left(\kappa_{\sigma}+\kappa_{1}^{d}\right)+\frac{\phi_{\sigma}^{2}}{2}\right) \sigma_{g, t}^{2}\right) \mathrm{d} t \\
& +\phi_{\sigma} \sigma_{g, t}^{t} \mathrm{~d} W_{t}^{c}+\left(A_{x}^{d} \sigma_{x}+\phi_{\sigma, x}\right) \mathrm{d} W_{t}^{x}+A_{\sigma}^{d} \sqrt{q_{t}} \mathrm{~d} W_{t}^{\sigma} \\
& +A_{q}^{d} \varphi_{q} \sqrt{q_{t}} \mathrm{~d} W_{t}^{q}+\sigma_{d} \mathrm{~d} W_{t}^{d} .
\end{aligned}
$$

For the rest of the Appendix B, we will consider the market return that follows the above dynamics.B.2

\section{B.2 VARIANCE PREMIUM}

The variability of the future asset price is determined by the integrated variance:

$$
\begin{aligned}
\mathrm{IV}_{t, t+N} \equiv \int_{\tau=t}^{t+N} \mathrm{~d}[\log S, \log S]_{\tau}= & \phi_{\sigma}^{2} \int_{\tau=t}^{t+N} \sigma_{g \tau}^{2} \mathrm{~d} \tau+\left(A_{\sigma}^{d, 2}+A_{q}^{d, 2} \varphi_{q}^{2}\right) \int_{\tau=t}^{t+N} q_{\tau} \mathrm{d} \tau \\
& +N\left(\sigma_{d}^{2}+\left(A_{x}^{d} \sigma_{x}+\phi_{\sigma, x}\right)^{2}\right)
\end{aligned}
$$

The variance risk premium by definition is given by the difference between the expected values of the integrated variance under the objective and risk-neutral measures:

$$
\mathrm{vp}_{t} \equiv E_{t}^{Q} \mathrm{IV}_{t, t+N}-E_{t}^{P} \mathrm{IV}_{t, t+N} .
$$

Under the objective measure, the consumption variance $\sigma_{g, t}^{2}$ and the volatility-ofvolatility $q_{t}$ are both affine processes with expectations:

$$
E_{t}^{P} q_{t+\Delta t}=\left[q_{t}-\mu_{q}\right] \mathrm{e}^{-\kappa_{q} \Delta t}+\mu_{q}
$$




$$
E_{t}^{P} \sigma_{g, t+\Delta t}^{2}=\left[\sigma_{g, t}^{2}-\mu_{\sigma}\right] \mathrm{e}^{-\kappa_{\sigma} \Delta t}+\mu_{\sigma} .
$$

Under the risk-neutral measure, the volatility-of-volatility $q_{t}$ remains an affine process, with the mean reversion $\tilde{\kappa}_{q}=\kappa_{q}-(\theta-1) A_{q} \varphi_{q}^{2}$ and the mean $\tilde{\mu}_{q}=\frac{\kappa_{q} \mu_{q}}{\tilde{\kappa}_{q}}$ given by Equation (B.11). Thus, the expectation of $q_{t}+\Delta t$ under the risk-neutral measure simply equals:

$$
E_{t}^{Q} q_{t+\Delta t}=\left[q_{t}-\tilde{\mu}_{q}\right] \mathrm{e}^{-\tilde{\kappa}_{q} \Delta t}+\tilde{\mu}_{q} .
$$

The process for the variance $\sigma_{g, t}^{2}$ under the risk-neutral measure in Equation (B.10) is qualitatively different. The conditional mean now depends not only on its own value but also on the current realization of $q_{t}$ :

$$
E_{t}^{Q} \sigma_{g t+\Delta t}=\tilde{\mu}_{\sigma}+\left(\sigma_{g, t}^{2}-\tilde{\mu}_{\sigma}-\Delta_{q}\right) \mathrm{e}^{-\kappa_{\sigma} \Delta t}+\Delta_{q} \mathrm{e}^{-\tilde{\kappa}_{q} \Delta t},
$$

where

$$
\Delta_{q}=\frac{(\theta-1) A_{\sigma}}{\kappa_{\sigma}-\tilde{\kappa}_{q}}\left[q_{t}-\tilde{\mu}_{q}\right]
$$

and $\tilde{\mu}_{\sigma}=\mu_{\sigma}+\frac{(\theta-1) A_{\sigma}}{\kappa_{\sigma}} \tilde{\mu}_{q}$ is equal to the risk-neutral unconditional mean of the variance.

The differences in the conditional expectations of the state variables under the risk-neutral and the objective measures can be represented as follows:

$$
\begin{aligned}
E_{t}^{Q} q_{t+\Delta t}-E_{t}^{P} q_{t}=q_{t}\left[\mathrm{e}^{-\tilde{\kappa}_{q} \Delta t}\right. & \left.-\mathrm{e}^{-\tilde{\kappa}_{q} \Delta t}\right]+\kappa_{q} \mu_{q}\left[\frac{1-\mathrm{e}^{-\tilde{\kappa}_{q}}}{\tilde{\kappa}_{q}}-\frac{1-\mathrm{e}^{-\kappa_{q} \Delta t}}{\kappa_{q}}\right], \\
\frac{E_{t}^{Q} \sigma_{g, t+\Delta t}^{2}-E_{t}^{P} \sigma_{g, t+\Delta t}^{2}}{(\theta-1) A_{\sigma}} & =q_{t}\left[\frac{\mathrm{e}^{-\tilde{\kappa}_{q} \Delta t}-\mathrm{e}^{-\kappa_{\sigma} \Delta t}}{\kappa_{\sigma}-\tilde{\kappa}_{q}}\right] \\
& +\tilde{\mu}_{q}\left[\frac{1-\mathrm{e}^{-\kappa_{\sigma} \Delta t}}{\kappa_{\sigma}}-\frac{\mathrm{e}^{-\tilde{\kappa}_{q} \Delta t}-\mathrm{e}^{-\kappa_{\sigma} \Delta t}}{\kappa_{\sigma}-\tilde{\kappa}_{q}}\right]
\end{aligned}
$$

Since $\exp (-x)$ and $(1-\exp (-x)) / x$ are both decreasing functions in $x$, it follows that $E_{t}^{Q} q_{t+\Delta t}>E_{t}^{P} q_{t}$ for $\tilde{\kappa}_{q}<\kappa_{q}\left(A_{q}<0\right)$. Similarly, it is possible to show that for any positive $\tilde{\kappa}_{q}, \kappa_{\sigma}$, and $\Delta t$, the expressions in square brackets in the second equation above are both greater than zero. Thus, the variance and volatility-of-volatility are both expected to be higher under the risk-neutral measure. Since the integrated variance in Equation (B.14) depends on future values of $q_{t}$ and $\sigma_{g, t}^{2}$, the variance premium $v p_{t} \equiv E_{t}^{Q} \mathrm{IV}_{t, t+N}-E_{t}^{P} \mathrm{IV}_{t, t+N}$ must be positive.

Going one step further, the variance premium may be expressed as follows: 


$$
v p_{t}=\beta_{p r, 0}+\beta_{p r, 1} q_{t},
$$

where

$$
\begin{aligned}
\beta_{p r, 0}= & \phi_{\sigma}^{2}\left(\tilde{\mu}_{\sigma}-\mu_{\sigma}\right)\left(N-\frac{1-\mathrm{e}^{-\kappa_{\sigma} N}}{\kappa_{\sigma}}\right)+\left(\tilde{\mu}_{q}-\mu_{q}\right)\left(A_{\sigma}^{d, 2}+A_{q}^{d, 2} \varphi_{q}^{2}\right)(N \\
& \left.-\frac{1-\mathrm{e}^{-\kappa_{q} N}}{\kappa_{q}}\right)-\beta_{p r, 1} \tilde{\mu}_{q}, \\
\beta_{p r, 1}= & \phi_{\sigma}^{2} \frac{(\theta-1) A_{\sigma}}{\kappa_{\sigma}-\tilde{\kappa}_{q}}\left(\frac{1-\mathrm{e}^{-\tilde{\kappa}_{q} N}}{\tilde{\kappa}_{q}}-\frac{1-\mathrm{e}^{-\kappa_{\sigma} N}}{\kappa_{\sigma}}\right)+\left(A_{\sigma}^{d, 2}+A_{q}^{d, 2} \varphi_{q}^{2}\right)\left(\frac{1-\mathrm{e}^{-\tilde{\kappa}_{q} N}}{\tilde{\kappa}_{q}}\right. \\
& \left.-\frac{1-\mathrm{e}^{-\kappa_{q} N}}{\kappa_{q}}\right) .
\end{aligned}
$$

The expression in Equation (B.15) is obtained by taking the difference between the expectations of the integrated variance under the objective measure,

$$
\begin{aligned}
E_{t}^{P} \operatorname{IV}_{t, t+N}= & \phi_{\sigma}^{2}\left(\mu_{\sigma} N+\frac{1-\mathrm{e}^{-\kappa_{\sigma} N}}{\kappa_{\sigma}}\left(\sigma_{g, t}^{2}-\mu_{\sigma}\right)\right)+\left(A_{\sigma}^{d, 2}+A_{q}^{d, 2} \varphi_{q}^{2}\right)\left(\mu_{q} N\right. \\
& \left.+\frac{1-\mathrm{e}^{-\kappa_{q} N}}{\kappa_{q}}\left(q_{t}-\mu_{q}\right)\right)+N\left(\sigma_{d}^{2}+\left(A_{x}^{d} \sigma_{x}+\phi_{\sigma,}^{x}\right)^{2}\right),
\end{aligned}
$$

and under the risk-neutral measure,

$$
E_{t}^{Q} \mathrm{IV}_{t, t+N}=\beta_{\mathrm{VIX}, 0}+\beta_{\mathrm{VIX}, \sigma} \sigma_{g, t}^{2}+\beta_{\mathrm{VIX}, q} q_{t}^{2},
$$

where

$$
\begin{gathered}
\beta_{\mathrm{VIX}, \sigma}=\phi_{\sigma}^{2} \frac{1-\mathrm{e}^{-\kappa_{\sigma} N}}{\kappa_{\sigma}}, \\
\beta_{\mathrm{VIX}, q}=\phi_{\sigma}^{2} \frac{(\theta-1) A_{\sigma}}{\kappa_{\sigma}-\tilde{\kappa}_{q}}\left[\frac{1-\mathrm{e}^{-\tilde{\kappa}_{q} N}}{\tilde{\kappa}_{q}}-\frac{1-\mathrm{e}^{-\kappa_{\sigma} N}}{\kappa_{\sigma}}\right]+\left(A_{\sigma}^{d, 2}+A_{q}^{2} \varphi_{q}^{d, 2}\right) \frac{1-\mathrm{e}^{-\tilde{\kappa}_{q} N}}{\tilde{\kappa}_{q}} \\
\beta_{\mathrm{VIX}, 0}=\left(\phi_{\sigma}^{2} \tilde{\mu}_{\sigma}+\left(A_{\sigma}^{d, 2}+A_{q}^{d, 2} \varphi_{q}^{2}\right) \tilde{\mu}_{q}+\sigma_{d}^{2}+\left(A_{x}^{d} \sigma_{x}+\phi_{\sigma, x}\right)^{2}\right) N-\beta_{\mathrm{VIX}, \sigma^{2}} \tilde{\mu}_{\sigma} \\
-\beta_{\mathrm{VIX}, q} \tilde{\mu}_{q} .
\end{gathered}
$$


As discussed further in the main text, the expectation under the risk-neutral measure corresponds directly to the VIX ${ }^{2}$ volatility index, hence the subscript notation for the $\beta \mathrm{s}$.

\section{B.3. RETURN-VOLATILITY CROSS-CORRELATIONS}

The return over a short time interval $\Delta t$ is approximately equal to:

$$
\begin{aligned}
\Delta \log R_{t}^{d} \approx & \left(\rho+\frac{\mu_{g}+x_{t}}{\psi}-A_{q}^{d}\left(\kappa_{q}+\kappa_{1}^{d}\right) q_{t}-\left(A_{\sigma}^{d}\left(\kappa_{\sigma}+\kappa_{1}^{d}\right)+\frac{\phi_{\sigma}^{2}}{2}\right) \sigma_{g, t}^{2}\right. \\
& \left.+\frac{\theta(\theta-1) A_{x}^{2} \sigma_{x}^{2}-\left((\theta-1) A_{x} \sigma_{x}+A_{x}^{d} \sigma_{x}+\phi_{\sigma, x}\right)^{2}}{2}-\frac{\sigma_{d}^{2}}{2}\right) \Delta t \\
& +\phi_{\sigma} \sigma_{g, t} \Delta W_{t}^{c}+\left(A_{x}^{d} \sigma_{x}+\phi_{\sigma, x}\right) \Delta W_{t}^{x}+A_{\sigma}^{d} \sqrt{q_{t}} \Delta W_{t}^{\sigma} \\
& +A_{q}^{d} \varphi_{q} \sqrt{q_{t}} \Delta W_{t}^{q}+\sigma_{d} \Delta W_{t}^{d},
\end{aligned}
$$

where the operator $\Delta$ denotes the increment to the process over the $[t, t+\Delta]$ time interval. The variance of the return equals:

$$
\begin{aligned}
\operatorname{Var}\left(\Delta \log R_{t}\right)= & \left(\beta_{R, \sigma}^{2} \operatorname{Var}\left(\sigma_{g, t}^{2}\right)+\beta_{R, q}^{2} \operatorname{Var} q_{t}+\frac{1}{\psi^{2}} \operatorname{Var} x_{t}\right)(\Delta t)^{2}+\mu_{\sigma} \phi_{\sigma}^{2} \Delta t \\
& +\left(A_{\sigma,}^{d, 2}+A_{q,}^{d, 2} \varphi_{q}^{2}\right) \mu_{q} \Delta t+\Delta t\left(\sigma_{d}^{2}+\left(A_{x}^{d} \sigma_{x}+\phi_{\sigma,}^{x}\right)^{2}\right)
\end{aligned}
$$

where $\beta_{R, \sigma}=-\left(A_{\sigma}^{d}\left(\kappa_{\sigma}+\kappa_{1}^{d}\right)+\frac{\phi_{\sigma}^{2}}{2}\right)$ and $\beta_{R, q}=-A_{q}^{d}\left(\kappa_{1}+\kappa_{q}^{d}\right)$.

From Equation (B.15), the variance premium is directly proportional to $q_{t}$. Hence, the correlation between the premium and the return is solely determined by the correlation of the return with the $q_{t}$ process. The covariance between $q_{t}$ and a future return, $\Delta \log R_{t+l}$ and $l>0$, is equal to the covariance of $q_{t}$ with the drift part of the return:

$$
\operatorname{Cov}\left(q_{t}, \Delta \log R_{t+l}\right)=\mathrm{e}^{-\kappa_{q} l} \operatorname{Var} q_{t} \beta_{R, q} \Delta t, \quad l \geq 0 .
$$

The covariance of $q_{t}$ with the past return, $\Delta \log R_{t-l}$ and $l<0$, consists of two parts. The covariance with the drift $\mathrm{e}^{-\kappa_{q} l} \operatorname{Var} q_{t} \beta_{R, q} \Delta t$ and the covariance with the diffusive part $A_{q}^{d} \varphi_{q} \operatorname{Cov}\left(q_{t}, \sqrt{q_{t-l}} \Delta W_{t-l}^{q}\right)=A_{q}^{d} \varphi_{q}^{2} \mu_{q} \mathrm{e}^{-\kappa_{q} l} \Delta t$. Combining these effects, the cross-correlation function for the variance risk premium and the returns may be conveniently expressed as follows:

$$
\operatorname{Corr}\left(q_{t}, \Delta \log R_{t+l}\right)=\frac{\operatorname{Var} q_{t} \beta_{R, q}+I_{l<0} A_{q}^{d} \varphi_{q}^{2} \mu_{q} \mathrm{e}^{-\kappa_{q}|l|} \Delta t}{\sqrt{\operatorname{Var} q_{t}} \sqrt{\operatorname{Var}\left(\Delta \log R_{t}\right)}}
$$

for any value of $l$. 
The expression for the $\mathrm{VIX}_{t}^{2} \equiv E_{t}^{Q} \mathrm{IV}_{t, t+N}$ in Equation (B.16) involves a linear function of $q_{t}$ and the variance $\sigma_{g, t}$, with loadings $\beta_{\mathrm{VIx}, q}$ and $\beta_{\mathrm{VIx}, \sigma}$, respectively. The covariance of the VIX ${ }_{t}^{2}$ with any future return depends solely on the covariance with the drift of the return:

$$
\operatorname{Cov}\left(\mathrm{VIX}_{t}^{2}, \Delta \log R_{t+l}\right)=\left(\beta_{R, \sigma} \beta_{\mathrm{VIX}, \sigma} \operatorname{Var} \sigma_{g, t}^{2} \mathrm{e}^{-\kappa_{\sigma} l}+\beta_{R, q} \beta_{\mathrm{VIX}, q} \operatorname{Var} q_{t} \mathrm{e}^{-\kappa_{q} l}\right) \Delta t .
$$

The covariance of the VIX ${ }_{t}^{2}$ with past returns includes the covariances with the drift and the diffusion:

$$
\begin{aligned}
\operatorname{Cov}\left(\operatorname{VIX}_{t}^{2}, \Delta \log R_{t-l}\right)= & \left(\left(\beta_{R, \sigma} \operatorname{Var} \sigma_{g, t}^{2}+A_{\sigma}^{d} \mu_{q}\right) \beta_{\mathrm{VIX}, \sigma} \mathrm{e}^{-k_{\sigma} l}+\left(\beta_{R, q} \operatorname{Var} q_{t}\right.\right. \\
& \left.\left.+A_{q}^{d} \varphi_{q}^{2} \mu_{q}\right) \beta_{\mathrm{VIX}, q} \mathrm{e}^{-\kappa_{q} l}\right) \Delta t .
\end{aligned}
$$

Combining these expressions, the cross-correlations $\operatorname{Corr}\left(\mathrm{VIX}_{t}^{2}, \Delta \log R_{t+l}\right)$ may be succinctly written as follows:

$$
\frac{\left(\beta_{R, \sigma} \operatorname{Var} \sigma_{g, t}^{2}+I_{l<0} A_{\sigma}^{d} \mu_{q}\right) \beta_{\mathrm{VIX}, \sigma} \mathrm{e}^{-\kappa_{\sigma}|l|} \Delta t+\left(\beta_{R, q} \operatorname{Var} q_{t}+I_{l<0} A_{q}^{d} \varphi_{q}^{2} \mu_{q}\right) \beta_{\mathrm{VIX}, q} \mathrm{e}^{-\kappa_{q}|l|} \Delta t}{\sqrt{\left(\beta_{\mathrm{VIX}, \sigma}^{2} \operatorname{Var} \sigma_{g, t}^{2}+\beta_{\mathrm{VIX}, q}^{2} \operatorname{Var} q_{t}\right) \operatorname{Var}\left(\Delta \log R_{t}\right)}}
$$

for any value of $l$.

\section{Appendix C: Calibration Details}

Table II shows the parameter values used in the calibration and their sources. Apart from the parameters governing the volatility dynamics, all the values are taken directly from the existing literature with a few small adjustments to ensure a well-defined model solution. In particular, the three preference parameters, $\rho, \gamma$, and $\psi$, are all in the generally agreed upon reasonable range and directly in line with the values used by Bansal and Yaron (2004). To allow for accurate log-linear approximations and internally consistent calibration results, we explicitly solve for the two log-linearization parameters $\kappa_{1}$ and $\kappa_{1}^{d}$ within the model. ${ }^{31}$

The actual calibration results reported in Table III generally reveal a close match between the model-implied and observed sample moments taken from the existing literature and well within acceptable accuracy tolerances traditionally used in the macrofinance literature. ${ }^{32}$ The only exception being the variability of the short rate.

\footnotetext{
31 For further discussion along these lines, see also Beeler and Campbell (2009).

32 The values of $\kappa_{1}=0.00052$ and $\kappa_{1}^{d}=0.0037$ solved for within the model and the corresponding implied annual price-dividend ratio of 22.61 also closely matches the 25.56 sample value reported in Bansal and Yaron (2004).
} 
The empirically low variability of the short rate presents a well-known difficulty for many equilibrium models. It is intimately related to the value of the intertemporal elasticity of substitution, or $\psi$, and the variance of $\sigma_{g, t}^{2}$, as discussed in more detail in Bansal and Yaron (2004).

The variability of the VIX ${ }^{2}$ and the variance risk premium are both extremely difficult, if not impossible, to reliable estimate with the limited time span of available data, and we purposely do not report any observed values for these statistics in the table. ${ }^{33}$ Nonetheless, the model-implied values of $\sqrt{\operatorname{Var}\left(\operatorname{VIX}_{t}^{2}\right)}=100.6$ and $\sqrt{\operatorname{Var}\left(v p_{t}\right)}=29.37$ are arguably both on the high side. This excess variability of the two variance measures stems from the assumption about the underlying economic uncertainty and the corresponding processes parameters, as formally discussed in Section 3.1. By assuming a short-memory process for $\sigma_{g, t}^{2}$, the calibration underestimates the persistence in $\mathrm{VIX}^{2}$ and in turn overestimates the model-implied variation. This is in part due to the fact that the two volatility factors, $\sigma_{g, t}^{2}$ and $q_{t}$, are assumed to be independent. This assumption facilitates a direct study of the two separate sources of risk. The drawback, however, is that the variance premium is significantly reduced relative to a model with correlated volatility risk, as in, for example, Drechsler and Yaron (2011), where the volatility-of-volatility is effectively an affine function of the variance. It is possible to alleviate this constraint within the basic model setup by allowing for correlated volatility shocks, that is, $\operatorname{Corr}\left(\mathrm{d} W_{t}^{q}, \mathrm{~d} W_{t}^{\sigma}\right)>0$. We purposely did not pursue that channel here to keep the two effects naturally separated. Alternatively, the model could be extended to incorporate non-Gaussian shocks, as in Drechsler and Yaron (2011) and Wachter (2010), to help break the algebraic constraints on the volatility of $q_{t}$ for a given volatility of $\sigma_{g, t}^{2}$. Such an extension would allow for a larger variance premium while keeping the overall volatility constant. The same modification could also help decrease the variability of the short rate, which increases with the variance of $\sigma_{g, t}^{2}$, but is not very sensitive to the variance of $q_{t}$.

Everything else equal, a more persistent $q_{t}$ process also increases the value of $A_{q}$ and, in turn, the sensitivity of the pricing kernel to innovations in $q_{t}$. However, it is important to note that even if $q_{t}$ was constant, a sufficiently persistent variance process could still generate a nontrivial variance risk premium within the model. Of course, the persistence of the $q_{t}$ process also plays an important role in determining the return predictability over longer horizons inherent in the variance risk premium recently highlighted by Bollerslev, Tauchen, and Zhou (2009). The additional

\footnotetext{
33 To illustrate, on estimating $\sqrt{\operatorname{Var}\left(V I X_{t}^{2}\right)}$ and $\sqrt{\operatorname{Var}\left(v p_{t}\right)}$ with data from 2007 through the end of 2008, the summary statistics reported in Table I increase from 23.70 to 93.60 and from 12.62 to 55.41, respectively.
} 
calibrations discussed in the supplementary material further illustrate this point and other longer run return predictability relations.

\section{Appendix D: General Model Solution}

In this section, we consider more flexible volatility dynamics and abstract from the difference between consumption and dividend assets. We assume that the process for the consumption variance has a general Moving Average (MA) representation:

$$
\sigma_{g, t}^{2}=\sigma^{2}+\int_{-\infty}^{t} a(t-s) \sqrt{q_{s}} \mathrm{~d} W_{s}^{\sigma} .
$$

This specification includes the short-memory model in Equation (B.3) above as a special case but importantly allows for much richer dynamic dependencies, including long-memory in which the $a(t-s)$ coefficients decrease at a slow hyperbolical rate. We maintain the identical short-memory process for the volatility-ofvolatility in Equation (B.4) but, for simplicity, focus only on the case of the constant expected consumption growth:

$$
\frac{\mathrm{d} C_{t}}{C_{t}}=\mu_{g} \mathrm{~d} t+\sigma_{g, t} \mathrm{~d} W_{t}^{c} .
$$

The pricing relation in Equation (B.5) remains the same. In parallel to the solution method for the short-memory model used above, the linearization of the price-dividend ratio reduces the problem to a system of linear equations. In general, all the shocks in Equation (D.1) need to be included in the conjectured solution for the dividend-price ratio:

$$
\log \Psi_{t}=A_{0}+A_{q} q_{t}+\int_{-\infty}^{t} A(t-s) \sqrt{q_{s}} \mathrm{~d} W_{s}^{\sigma} .
$$

Following Rogers (1997), if the price is a semimartingale, as it must be to prevent arbitrage, and $A(t)$ exists and is differentiable at zero, the dynamics of the pricedividend ratio may be decomposed into separate drift and diffusion terms:

$$
\begin{aligned}
\operatorname{d} \log \Psi_{t}= & {\left[A_{q} \kappa_{q}\left(\mu_{q}-q_{t}\right)+\int_{-\infty}^{t} A^{\prime}(t-s) \sqrt{q_{s}} \mathrm{~d} W_{s}^{\sigma}\right] \mathrm{d} t+A_{q} \varphi_{q} \sqrt{q_{t}} \mathrm{~d} W_{t}^{q} } \\
& +A(0) \sqrt{q_{t}} \mathrm{~d} W_{t}^{\sigma} .
\end{aligned}
$$


Now, substituting the conjectured solution into the pricing equation imply that the loadings on the variance shocks must satisfy:

$$
A^{\prime}(t-s)-\kappa_{1} A(t-s)=\frac{\gamma\left(1-\frac{1}{\psi}\right)}{2} a(t-s)
$$

for all $t \geq s$. Solving this system along with the constant and the loading on $q_{t}$ in Equation (D.2), we obtain the following:

$$
\begin{aligned}
& A(t)=-\int_{t}^{+\infty} \frac{\gamma\left(1-\frac{1}{\psi}\right)}{2} \mathrm{e}^{\kappa_{1}(t-\tau)} a(\tau) \mathrm{d} \tau \\
& A_{q}=\frac{\kappa_{q}+\kappa_{1}-\sqrt{\left(\kappa_{q}+\kappa_{1}\right)^{2}-\theta^{2} \varphi_{q}^{2} A(0)^{2}}}{\theta \varphi_{q}^{2}}, \\
& A_{0}=\frac{A_{q} \kappa_{q} \mu_{q}-\kappa_{0}+\left(1-\frac{1}{\psi}\right) \mu_{g}-\rho-\frac{\gamma}{2}\left(1-\frac{1}{\psi}\right) \sigma^{2}}{\kappa_{1}} .
\end{aligned}
$$

If absolute values of the coefficients $a(t)$ are decreasing in $t$ (or grow at a rate less than exponential) and $|a(t)|<\infty$, then $A(t)$ is well defined and $A \prime(0)=$ $\kappa_{1} A(0)+\frac{\gamma\left(1-\frac{-}{\psi}\right)}{2} a(0)$ is finite.

Substituting the solution for the price-dividend ratio into the expression for the $\mathrm{SDF}$, it follows that

$$
\begin{aligned}
\frac{\mathrm{d} M_{t}}{M_{t}}= & {\left[-\frac{\mu_{g}}{\psi}-\rho+\frac{\gamma}{2}\left(1+\frac{1}{\psi}\right) \sigma_{g, t}^{2}+\left(\frac{1}{\theta}-1\right)\left(\kappa_{1}+\kappa_{q}\right) A_{q} q_{t}\right] \mathrm{d} t } \\
& -\gamma \sigma_{g, t} \mathrm{~d} W_{t}^{c}+(\theta-1) A_{q} \varphi_{q} \sqrt{q_{t}} \mathrm{~d} W_{t}^{q}+(\theta-1) A(0) \sqrt{q_{t}} \mathrm{~d} W_{t}^{\sigma} .
\end{aligned}
$$

As before, the risk-free rate is simply defined by the drift of $M_{t}$ :

$$
r_{t}^{r f}=-E_{t} \frac{\mathrm{d} M_{t}}{M_{t} \mathrm{~d} t}=\frac{\mu_{g}}{\psi}+\rho-\frac{\gamma}{2}\left(1+\frac{1}{\psi}\right) \sigma_{g, t}^{2}+\left(1-\frac{1}{\theta}\right)\left(\kappa_{1}+\kappa_{q}\right) A_{q} q_{t} .
$$

Since $\mathrm{d} \log R_{t}=\mathrm{d} \log C_{t}+\mathrm{d} \log \Psi_{t}+\Psi_{t}^{-1} \mathrm{~d} t$, and therefore,

$$
\mathrm{d} \log R_{t}=D \log R_{t} \mathrm{~d} t+\sigma_{g, t} \mathrm{~d} W_{t}^{c}+A_{q} \varphi_{q} \sqrt{q_{t}} \mathrm{~d} W_{t}^{q}+A(0) \sqrt{q_{t}} \mathrm{~d} W_{t}^{\sigma} .
$$

The equity premium for the consumption asset equals: 
$\pi_{r, t}=\gamma \sigma_{g, t}^{2}+(1-\theta)\left[A_{q}^{2} \varphi_{q}^{2}+A(0)^{2}\right] q_{t}=\gamma \sigma_{g, t}^{2}+2\left(\frac{1}{\theta}-1\right)\left(\kappa_{q}+\kappa_{1}\right) A_{q} q_{t}$

And, the dynamics of the return is determined by

$$
\begin{aligned}
\mathrm{d} \log R_{t}= & {\left[\rho+\frac{\mu_{g}}{\psi}+\left[-\frac{1}{2}+\frac{\gamma}{2}\left(1-\frac{1}{\psi}\right)\right] \sigma_{g, t}^{2}-\left(\kappa_{q}+\kappa_{1}\right) A_{q} q_{t}\right] \mathrm{d} t } \\
& +\sigma_{g, t} \mathrm{~d} W_{t}^{c}+A_{q} \varphi_{q} \sqrt{q_{t}} \mathrm{~d} W_{t}^{q}+A(0) \sqrt{q_{t}} \mathrm{~d} W_{t}^{\sigma} .
\end{aligned}
$$

The integrated variance may generally be expressed as follows:

$$
\mathrm{IV}_{t, t+N}=\int_{t}^{t+N} \sigma_{g, \tau}^{2} \mathrm{~d} \tau+\left(A_{q}^{2} \varphi_{q}^{2}+A(0)^{2}\right) \int_{t}^{t+N} q_{\tau} \mathrm{d} \tau .
$$

The expected value of the integrated variance under the objective measure equals:

$$
\begin{aligned}
E_{t}^{P} \mathrm{IV}_{t, t+N}= & \sigma^{2} N+\int_{-\infty}^{t}\left[\int_{t}^{t+N} a(\tau-s) \mathrm{d} \tau\right] \sqrt{q_{s}} \mathrm{~d} W_{s}^{\sigma}+\left(A_{q}^{2} \varphi_{q}^{2}+A(0)^{2}\right)\left[\mu_{q} N\right. \\
& \left.+\frac{1-\mathrm{e}^{-\kappa_{q} N}}{\kappa_{q}}\left(q_{t}-\mu_{q}\right)\right]
\end{aligned}
$$

The expectation under the risk-neutral measure is as follows:

$$
\begin{aligned}
E_{t}^{Q} \mathrm{IV}_{t, t+N} & =\sigma^{2} N+\int_{-\infty}^{t} \int_{t}^{t+N} a(\tau-s) \sqrt{q_{s}} \mathrm{~d} \tau \mathrm{d} W_{s}^{\sigma} \\
& +E_{t}^{Q} \int_{t}^{t+N} \int_{t}^{\tau} a(\tau-s) \sqrt{q_{s}} \mathrm{~d} W_{s}^{\sigma} \mathrm{d} \tau+\left(A_{q}^{2} \varphi_{q}^{2}+A(0)^{2}\right)\left(\tilde{\mu}_{q} N\right. \\
& \left.+\frac{1-\mathrm{e}^{-\tilde{\kappa}_{q} N}}{\tilde{\kappa}_{q}}\left(q_{t}-\tilde{\mu}_{q}\right)\right),
\end{aligned}
$$

where $\tilde{\kappa}_{q}=\kappa_{q}-(\theta-1) A_{q} \varphi_{q}^{2}$ refers to the mean reversion of $q_{t}$ under the riskneutral probability and $\tilde{\mu}_{q}=\kappa_{q} / \tilde{\kappa}_{q} \mu_{q}$ denotes the corresponding expectation. Moreover, under the risk-neutral measure: 


$$
\begin{aligned}
E_{t}^{Q} \sqrt{q_{s}} \mathrm{~d} W_{s}^{\sigma} & =E_{t}^{Q}\left(\sqrt{q_{s}} \mathrm{~d} \tilde{W}_{s}^{\sigma}+(\theta-1) A(0) q_{s} \mathrm{~d} s\right) \\
& =(\theta-1) A(0)\left(\tilde{\mu}_{q}+\mathrm{e}^{-\tilde{\kappa}_{q}(s-t)}\left(q_{t}-\tilde{\mu}_{q}\right)\right) \mathrm{d} s
\end{aligned}
$$

Consequently, the variance risk premium defined by the difference between $E_{t}^{Q} \mathrm{IV}_{t, t+N}$ and $E_{t}^{P} \mathrm{IV}_{t, t+N}$ is again a linear function of $q_{t}$

$$
\mathrm{vp}_{t}=\beta_{p r, 0}+\beta_{p r, 1} q_{t}
$$

with the two coefficients now defined by

$$
\begin{gathered}
\beta_{p r, 0}=(\theta-1) A(0) \tilde{\mu}_{q} \int_{t}^{t+N} \int_{t}^{\tau} a(\tau-s)\left(1-\mathrm{e}^{-\tilde{\kappa}_{q}(s-t)}\right) \mathrm{d} s \mathrm{~d} \tau+\left(A_{q}^{2} \varphi_{q}^{2}\right. \\
\left.+A(0)^{2}\right)\left[\tilde{\mu}_{q}\left(N-\frac{1-\mathrm{e}^{-\tilde{\kappa}_{q} N}}{\tilde{\kappa}_{q}}\right)-\mu_{q}\left(N-\frac{1-\mathrm{e}^{-\kappa_{q} N}}{\kappa_{q}}\right)\right] \\
\beta_{p r, 1}=(\theta-1) A(0) \int_{t}^{t+N} \int_{t}^{\tau} a(\tau-s) \mathrm{e}^{-\tilde{\kappa}_{q}(s-t)} \mathrm{d} s \mathrm{~d} \tau \\
+\left(A_{q}^{2} \varphi_{q}^{2}+A(0)^{2}\right)\left[\frac{1-\mathrm{e}^{-\tilde{\kappa}_{q} N}}{\tilde{\kappa}_{q}}-\frac{1-\mathrm{e}^{-\kappa_{q} N}}{\kappa_{q}}\right]
\end{gathered}
$$

\section{References}

Aït-Sahalia, Y. and Lo, A. W. (2000) Nonparametric risk management and implied risk aversion, Journal of Econometrics 94, 9-51.

Andersen, T. G., Bollerslev, T., and Diebold, F. X. (2007) Roughing it up: including jump components in the measurement, modeling, and forecasting of return volatility, Review of Economics and Statistics 89, 701-720.

Andersen, T. G., Bollerslev, T., and Diebold, F. X. (2009) Parametric and nonparametric volatility measurement, in Y. Aït-Sahalia and L.P. Hansen, (eds.), Handbook of Financial Econometrics. Vol. 1, Elsevier Science, Amsterdam, The Netherlands, pp. 67-138.

Andersen, T. G., Bollerslev, T., , Diebold, F. X., and Labys, P. (2003) Modeling and forecasting realized volatility, Econometrica 71, 579-625.

Andersen, T. G., Bollerslev, T., , Diebold, F. X., and Vega, C. (2007) Real-time price discovery in global stock, bond and foreign exchange markets, Journal of International Economics 73, 251-277.

Andersen, T. G., Bollerslev, T., and Meddahi, N. (2004) Analytical evaluation of volatility forecasts, International Economic Review 45, 1079-1110. 
Baillie, R. T., Bollerslev, T., and Mikkelsen, H.-O. (1996) Fractionally integrated generalized autoregressive conditional heteroskedasticity, Journal of Econometrics 74, 3-30.

Bandi, F. and Perron, B. (2006) Long memory and the relation between implied and realized volatility, Journal of Financial Econometrics 4, 636-670.

Bansal, R. and Yaron, A. (2004) Risks for the long run: a potential resolution of asset pricing puzzles,, Journal of Finance 59, 1481-1509.

Barndorff-Nielsen, O. E., Hansen, P. R., , Lunde, A., and Shephard, N. (2008) Designing realised kernels to measure the ex-post variation of equity prices in the presence of noise, Econometrica 76, 1481-1536.

Beeler, J. and Campbell, J. Y. (2009) The long-run risk model and aggregate asset prices: an empirical assessment. Working Paper, Department of Economics, Harvard University.

Bekaert, G., Engstrom, E., and Xing, Y. (2009) Risk, uncertainty and asset prices, Journal of Financial Economics 91, 59-82.

Benzoni, L., Collin-Dufresne, P., and Goldstein, R. S. (2006) Can standard preferences explain the prices of out-of-the-money $S \& P 500$ put options?. NBER Working Paper No. 11861, University of Minnesota.

Black, F. (1976) Studies in stock price volatility changes. in: Proceedings from the American Statistical Association, Business and Economics Statistics Section, pp. 177-181.

Bollerslev, T., Gibson, M., and Zhou, H. (2011) Dynamic estimation of volatility risk premia and investor risk aversion from option-implied and realized volatilities, Journal of Econometrics 160, 235-245.

Bollerslev, T., Litvinova, L., and Tauchen, G. (2006) Leverage and volatility feedback effects in highfrequency data, Journal of Financial Econometrics 4, 353-384.

Bollerslev, T., Tauchen, G., and Zhou, H. (2009) Expected stock returns and variance risk premia, Review of Financial Studies 22, 4463-4492.

Bollerslev, T. and Zhou, H. (2006) Volatility puzzles: a simple framework for gauging returnvolatility regressions, Journal of Econometrics 131, 123-150.

Britten-Jones, M. and Neuberger, A. (2000) Option prices, implied price processes, and stochastic volatility, Journal of Finance 55, 839-866.

Calvet, L. E. and Fisher, A. J. (2002) Multifractality in asset returns: theory and evidence, Review of Economics and Statistics 84, 381-406.

Calvet, L. E. and Fisher, A. J. (2007) Multifrequency news and stock returns, Journal of Financial Economics 86, 178-212.

Calvet, L. E. and Fisher, A. J. (2008) Multifrequency jump-diffusions: an equilibrium approach, Journal of Mathematical Economics 44, 207-226.

Campbell, J. Y. (1996) Understanding risk and return, Journal of Political Economy 104, 298-345. Campbell, J. Y. and Hentschell, L. (1992) No news is good news: an asymmetric model of changing volatility in stock returns, Journal of Financial Economics 31, 281-318.

Campbell, J. Y., Lo, A. W., and MacKinlay, A. C. (1997) The Econometrics of Financial Markets, Princeton University Press, Princeton, New Jersey.

Campbell, J. Y. and Mankiw, G. (1989) Consumption, income and interest rates: reinterpreting the time series evidence, NBER Macro Annual 4, 185-216.

Campbell, J. Y. and Viceira, L. M. (2002) Strategic Asset Allocation: Portfolio Choice for Long-Term Investors. Oxford University Press, Oxford, United Kingdom.

Carr, P. and Madan, D. (1998) Towards a theory of volatility trading, in: R. Jarrow (ed.), Volatility: New Estimation Techniques for Pricing Derivatives. Risk Books, London, pp. 417-427.

Christie, A. A. (1982) The stochastic behavior of common stock variances: value, leverage and interest rate effects, Journal of Financial Economics 10, 407-432. 
Comte, F. and Renault, E. (1996) Long memory continuous time models, Journal of Econometrics 73, $101-149$.

Corsi, F. (2009) A simple long memory model of realized volatility, Journal of Financial Econometrics 7, 174-196.

Ding, Z., Grange, C. W., and Engle, R. F. (1993) A long memory property of stock market returns and a new model, Journal of Empirical Finance 1, 83-106.

Drechsler, I. and Yaron, A. (2011) What's vol got to do with it?, Review of Financial Studies 24, 1-45. Duffie, D. and Epstein, L. (1992a) Asset pricing with stochastic differential utility, Review of Financial Studies 5, 411-436.

Duffie, D. and Epstein, L. (1992b) Stochastic differential utility, Econometrica 60, 353-394.

Epstein, L. G. and Zin, S. E. (1991) Substitution, risk aversion, and the temporal behavior of consumption and asset returns: an empirical analysis, Journal of Political Economy 99, 263-286.

Eraker, B. (2008) The volatility premium, Working Paper, Department of Finance, University of Wisconsin.

Eraker, B. and Shaliastovich, I. (2008) An equilibrium guide to designing affine pricing models, Mathematical Finance 18, 519-543.

Figlewski, S. and Wang, X. (2002) Is the 'leverage effect' a leverage effect? , Working Paper, Stern School of Business, New York University.

French, K. F., Schwert, G. W., and Stambaugh, R. (1987) Expected stock returns and volatility, Journal of Financial Economics 19, 3-29.

Gabaix, X. (2010) Variable rare disasters: an exactly solved framework for ten puzzles in macrofinance, Working Paper, Stern School of Business, New York University.

Glosten, L. R., Jagannathan, R., and Runkle, D. E. (1993) On the relation between the expected value and the volatility of the nominal excess return on stocks, Journal of Finance 48, 1779-1801.

Gordon, S. and St-Amour, P. (2004) Asset returns and state-dependent risk preferences, Journal of Business and Economic Statistics 22, 241-252.

Hall, R. E. (1988) Intertemporal substitution in consumption, Journal of Political Economy 96, 221-273.

Hansen, P. R. and Lunde, A. (2010) Estimating the persistence and the autocorrelation function of a time series that is measured with error, Working Paper, Aarhus University, School of Economics and Management, and CREATES.

Jiang, G. J. and Tian, Y. S. (2005) Model-free implied volatility and its information content, Review of Financial Studies 18, 1305-1342.

Jiang, G. J. and Tian, Y. S. (2007) Extracting model-free volatility from option prices: an examination of the vix index, Journal of Derivatives 14, 1-26.

Kiku, D. (2008) Is the value premium a puzzle? Working Paper, Wharton School of Business, University of Pennsylvania.

Lettau, M., Ludvigson, S. C., and Wachter, J. A. (2009) The declining equity premium: what role does macroeconomic risk play?, Review of Financial Studies 21, 1653-1687.

Merton, R. C. (1973) An intertemporal capital asset pricing model, Econometrica 41, 867-887.

Nelson, D. B. (1991) Conditional heteroskedasticity in asset returns: a new approach, Econometrica 59, 347-370.

Nielsen, M. O. (2007) Local whittle analysis of stationary fractional cointegration and the impliedrealized volatility relation, Journal of Business and Economic Statistics 25, 427-446.

Robinson, P. (1991) Testing for strong serial correlation and dynamic conditional heteroskedasticity in multiple regression, Journal of Econometrics 47, 67-84.

Rogers, C. (1997) Arbitrage with fractional Brownian motion, Mathematical Finance 7, 95-105. 
Santa-Clara, P. and Yan, S. (2010) Crashes, volatility, and the equity premium: lessons from S\&P 500 options, Review of Economics and Statistics 92, 435-451.

Schwert, G. W. (1990) Stock volatility and the crash of '87, Review of Financial Studies 3, 77-102. Sizova, N. (2009) Integrated variance forecasting: model-based versus reduced form, Working Paper, Department of Economics, Rice University.

Vanden, J. M. (2005) Equilibrium analysis of volatility clustering, Journal of Empirical Finance 12, 374-417.

Wachter, J. A. (2010) Can time-varying risk of rare disasters explain aggregate stock market volatility? Working Paper, Wharton School of Business, University of Pennsylvania.

Zhang, L., Mykland, P. A., and Aït-Sahalia, Y. (2005) A tale of two time scales: determining integrated volatility with noisy high-frequency data, Journal of the American Statistical Association 100, 1394-1411. 